\title{
THE RESPONSE OF FRAMED BUILDINGS ON RAFT FOUNDATIONS TO TUNNELING
}

\author{
Jingmin $\mathrm{Xu}^{1}$, Andrea Franza ${ }^{2}$, and Alec M. Marshall ${ }^{3}$ \\ ${ }^{1} \mathrm{PhD}$ student, Department of Civil Engineering, University of Nottingham, Nottingham, UK. \\ Corresponding Author, Email: jingmin.xu@ nottingham.ac.uk \\ ${ }^{2}$ Research fellow, Universidad Politécnica de Madrid, Madrid, Spain. \\ ${ }^{3}$ Associate Professor, Department of Civil Engineering, University of Nottingham, Nottingham, \\ UK.
}

\begin{abstract}
This paper investigates the response of framed buildings on raft foundations to tunnel construction using geotechnical centrifuge testing. Five framed building models were considered and the influence of building configuration, weight, eccentricity, and soil density were evaluated. Soil and foundation displacements, frame deformed shape, maximum structure deformation parameters (deflection ratios and angular distortions), and associated modification factors are illustrated. Results indicate that, unlike equivalent isotropic plates, framed buildings primarily exhibit shear behavior and a semi-flexible response. Building deformed shapes indicate that angular/shear distortions (considering bay slope and local tilt) are more appropriate for quantifying framed building distortions than deflection ratios. A relative stiffness parameter is suggested to relate maximum angular distortions to greenfield settlement slope. Moreover, the efficiency of available relative stiffness parameters for the deflection ratio modification factors is confirmed. Limitations of the equivalent plate approach and practical implications of the results for framed buildings are discussed.
\end{abstract}




\section{INTRODUCTION}

Tunnels are constructed beneath congested urban areas to satisfy needs for new infrastructure. Associated with these underground construction activities is the requirement to evaluate the potential for damage to existing buildings that are subjected to tunneling-induced ground deformations.

The response of buildings to tunneling has been investigated extensively. While several studies have indicated the importance of considering realistic (framed) building characteristics (Boone, 1996; Boldini et al., 2018; Fu et al., 2018; Franza and DeJong, 2019; Son, 2015), equivalent beam or plate models are still generally adopted (Franzius et al., 2006; Pickhaver et al., 2010; Maleki et al., 2011; Namazi and Mohamad, 2013; Farrell et al., 2014; Bilotta et al., 2017; Franza and Marshall, 2018). This simplification involves uncertainties that have not yet been sufficiently investigated for framed buildings. When estimating the total structure stiffness, most researchers have adopted a pure bending stiffness (Franzius et al., 2006; Goh and Mair, 2014; Haji et al., 2018) despite the evidence that shear flexibility can have an important role (Potts and Addenbrooke, 1997; Finno et al., 2005). Also, Franza et al. (2020) highlighted that bending and shear deformation modes of equivalent solids have not always been well distinguished because of the focus on the structure settlement profile. Finally, the local deformations of infill walls in frames is potentially not well described by equivalent beams or plates (Goh and Mair, 2014; Boldini et al., 2018).

The tunnel-framed building interaction problem has frequently been studied using numerical modeling methods. Using the finite element method, Goh and Mair (2014) investigated the response of a framed structure on either continuous or individual footings to tunnel excavation, focusing on the influence of the frame action on building deflections. Son (2015) considered the distortion and cracking of two different framed structures subjected to tunneling in sandy ground, in which tunneling-related parameters and ground condition were varied. Boldini et al. (2018) conducted a parametric study of the response of reinforced concrete framed structures to tunneling using the finite element method and found that framed structures always display hogging zones at the ends of the foundation due to the non-uniform contract pressure at the soil-foundation interface.

There is a lack of experimental evidence to substantiate many of the findings reported by 
the above numerical analyses. The aim of this paper is to investigate, through reduced-scale centrifuge tests, how a framed building responds to tunneling-induced ground movements in terms of foundation and superstructure displacements. The paper includes results from 24 plane-strain geotechnical centrifuge tests designed to model tunneling induced ground displacements in dry sand and its effect on multi-story framed buildings with different characteristics and locations. Soil and building displacements were measured and, subsequently, used to relate bay and panel distortions to greenfield settlements. Limitations associated with the simplification of frames as equivalent isotropic plates are also illustrated.

\section{BACKGROUND}

\section{Risk assessment}

Excavation-induced ground movements can result in building displacements associated with both shear and bending deformations, as well as tilt (illustrated in Figure 1). In practice, structure deformations are generally estimated using a staged procedure (Mair et al., 1996): in the first (conservative) stage, greenfield movements are imposed on the building; if this results in an unacceptable risk level, then a second stage is conducted in which soil-structure interaction analyses are performed. To link building deformations with damage, the critical strain method is generally adopted (Boscardin and Cording, 1989); alternatively, thresholds from Son and Cording (2005) may be used. This associates the maximum tensile strain $\left(\epsilon_{\max }\right)$ within either bays or panels (defined in Figure 2), or the sagging/hogging portions of a building (see Figure 1) to a category of damage, as summarized in Table 1. Tensile strains are obtained using Equation (1) or (2) (Mair et al., 1996), based on a Mohr's circle of strain for a plane-stress or plane-strain condition, respectively, where $\epsilon_{x x}$ is longitudinal strain due to axial and bending deformations associated with curvature $\chi, \epsilon_{x z}$ is diagonal strain due to shear deformations associated with shear strain $\gamma$, and $v$ is Poisson's ratio.

$$
\begin{gathered}
\epsilon_{\max }=\frac{\epsilon_{x x}(1-v)}{2}+\sqrt{\frac{1}{4} \epsilon_{x x}^{2}(1+v)^{2}+\epsilon_{x z}^{2}} \\
\epsilon_{\max }=\frac{\epsilon_{x x}}{2}+\sqrt{\frac{1}{4} \epsilon_{x x}^{2}+\epsilon_{x z}^{2}}
\end{gathered}
$$


TABLE 1. Critical tensile strain and categories of damage (Boscardin and Cording, 1989).

\begin{tabular}{ccc}
\hline $\begin{array}{c}\text { Category } \\
\text { of damage }\end{array}$ & $\begin{array}{c}\text { Level } \\
\text { of damage }\end{array}$ & $\begin{array}{c}\text { Limiting tensile } \\
\text { strain }(\%)\end{array}$ \\
\hline 0 & Negligible & $0-0.05$ \\
1 & Very slight & $0.05-0.075$ \\
2 & Slight & $0.075-0.15$ \\
3 to 4 & Moderate to severe & $0.15-0.3$ \\
4 to 5 & Severe to very severe & $>0.3$ \\
\hline
\end{tabular}

Traditionally, longitudinal and diagonal strains are evaluated from the vertical and horizontal building displacements at the ground surface level (i.e. the bottom of the structure, see Figure 1), from which horizontal strains are inferred. Following this, sagging and hogging deflection ratios $D R_{\text {sag/hog }}$ can be inferred from the maximum deflections $\Delta_{\text {sag/hog }}$ and lengths of the sagging/hogging zones $B_{\text {sag } / \text { hog }}$ (Mair et al., 1996); or angular distortion $\beta$ of each bay can be obtained from the total building rotation $\omega^{\prime}$ and the slope $S$ of the settlement profile (Boscardin and Cording, 1989). Note in Figure 1 the difference between the building total tilt $\omega^{\prime}$ and the local tilt $w$, which is the cross-sectional rotation.

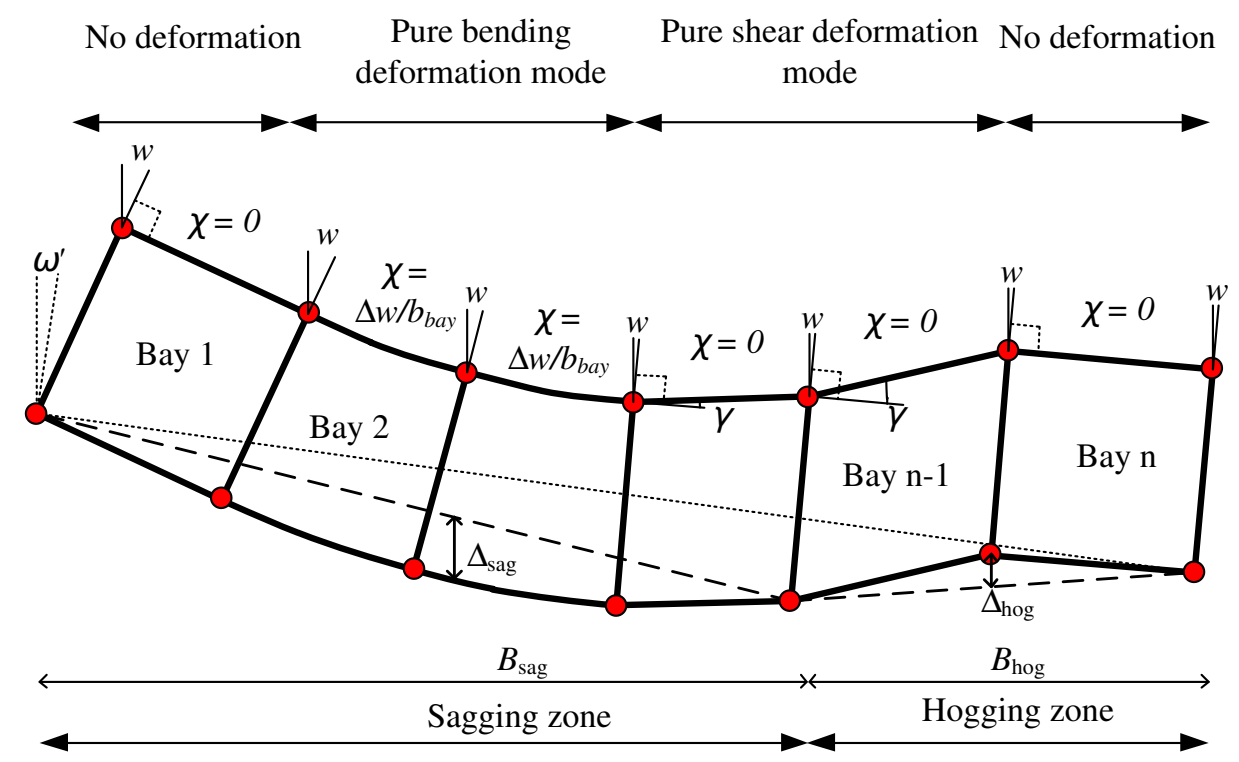

Fig. 1. Distortions of a building due to settlements and illustration of deformation parameters.

\section{Deflection ratios, equivalent stiffness, and relative stiffness parameters}

To characterize the effects of building stiffness on the soil-structure interaction, Potts and Addenbrooke (1997) introduced the deflection ratio modification factors $M^{D R, s a g}$ and $M^{D R, h o g}$, 
defined as:

$$
M^{D R, s a g / h o g}=\frac{D R_{\text {sag } / \text { hog, bld }}}{D R_{\text {sag } / \text { hog }, g f}}
$$

where $D R_{\text {sag/hog,bld }}$ and $D R_{\text {sag/hog,gf }}$ are, respectively, the deflection ratios of the building settlement profile and the greenfield settlement trough.

These modification factors have been related to relative soil-structure stiffness in a variety of formats (e.g. Potts and Addenbrooke (1997); Franzius et al. (2006)); in particular, Mair (2013) proposed the relative bending stiffness factors $\rho_{\text {sag } / \text { hog }}$ as:

$$
\rho_{\text {sag } / \text { hog }}=\frac{E I}{E_{s} B_{\text {sag } / \text { hog, } g f}^{3} L}=\frac{E I^{*}}{E_{s} B_{\text {sag } / \text { hog,gf }}^{3}}
$$

where $E I\left(\mathrm{kN} \mathrm{m}^{2}\right)$ is the bending stiffness of the building, $E I^{*}\left(\mathrm{kN} \mathrm{m}^{2} / \mathrm{m}\right)$ is $E I$ per running meter of the building, $E_{S}$ is the representative Young's modulus of the soil, $B_{\text {sag/hog,g } f}$ is the length of building in the sagging/hogging zones defined by the greenfield settlement inflection point, and $L$ is the length of the building in the longitudinal direction of the tunnel. In Equation (4), $E I^{*}$ is used to indicate the total building stiffness (e.g. Goh and Mair (2014); Haji et al. (2018)) without distinguishing between bending and shear contributions.

To better represent the stiffness effects, Pickhaver et al. (2010) and Finno et al. (2005) suggested that buildings could be modelled with equivalent Timoshenko and laminated beams with both cross-sectional bending stiffness $E I$ and shear stiffness $G A_{s}$, whereas Losacco et al. (2016) used anisotropic equivalent solids. To isolate the contributions of shear and bending stiffness, Franza et al. (2020) modified the relative stiffness parameter $\eta$ from Timoshenko beam theory as:

$$
\begin{array}{r}
\eta_{\text {sag } / \text { hog }}=\frac{\rho_{\text {sag } / \text { hog }}}{1+a F_{\text {sag } / \text { hog }}}=\frac{E I^{*}}{E_{S} B_{\text {sag } / \text { hog }, g}^{3}\left(1+a F_{\text {sag } / \text { hog }}\right)} \\
F_{\text {sag } / \text { hog }}=\frac{E I}{B_{\text {sag } / \text { hog }, g f}^{2} G A_{s}}
\end{array}
$$

where $a F=\delta_{\text {shear }} / \delta_{\text {bending }}$ is the ratio between shear $\left(\delta_{\text {shear }}\right)$ and bending $\left(\delta_{\text {bending }}\right)$ deflections, $F$ is a dimensionless factor, and $a=12$ was estimated from a simply supported beam condition. In general, $F<1 / 25$ results in bending-dominated behavior, whereas $F>1$ indicates sheardominated behavior. 


\section{Angular distortion and shear strains}

Recent studies have linked measured or computed building displacements at varying elevations to the deformation parameters. For instance, for the tunnel-building interaction problem, Franza et al. (2020) suggested the Direct Strain Based Approach in which $\chi$ and $\gamma$ estimated from bay displacements are input into Equations (1) and (2) to directly evaluate $\epsilon_{\max }$, with no need to partition the building into sagging and hogging zones.

Several approaches are available to evaluate deformation parameters from building displacements. Son and Cording (2005) directly estimated the angular distortion $\beta$ using the top and bottom corner displacements of each bay (i.e. from the local cross-sectional rotations $w$ ) by subtracting the tilt $w$ from the slope $S$, as described by:

$$
\beta=S-w=\frac{U_{z, D}-U_{z, C}}{b_{b a y}}-\frac{U_{x, A}-U_{x, C}}{H}
$$

where for $U_{i, j}, i=x ; z$ is the displacement direction, and $j=A ; B ; C ; D$ is the location of the bay corner; as shown in Figure 2(a), C and D are the two lower corners of the base whereas A and B are the upper corners of an $n$-story framed building; $H$ is the total height of the building; and $b_{\text {bay }}$ is the bay width.

To isolate tilt, bending displacements, and shear displacements, Cook (1994) also suggested to use the top and bottom corner displacements of each bay. This approach was used on centrifuge test data by Ritter et al. (2020) and Franza et al. (2020) to evaluate bay curvature $\chi$ and shear strain $\gamma$ from bending and shear displacements of bays (see Figure 1). In particular, Ritter et al. (2020) derived Equation (7) for the calculation of average shear distortion $\gamma$ within the considered bay. Importantly, Ritter et al. (2020) highlighted that $\beta \approx \gamma$ for the methods of Son and Cording (2005) and Cook (1994) (e.g. they would match for a Timoshenko beam).

$$
\gamma=\frac{U_{z, D}-U_{z, C}}{b_{\text {bay }}}-\frac{3 U_{x, A}-3 U_{x, C}-\left(U_{x, B}-U_{x, D}\right)}{2 H}
$$

Finally, several studies have distinguished between the shear deformations within a bay (averaged along the full-height of the building, as in Son and Cording (2005) and Cook (1994)), and the panel distortions (Boone, 1996; Elkayam and Klar, 2019). For example, for evaluating the distortions of 


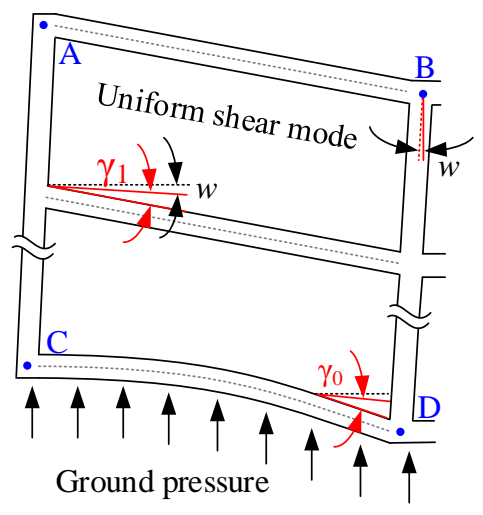

(a) Hinged end beam

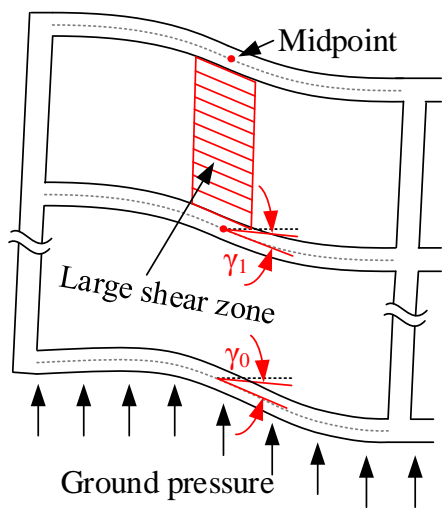

(b) Fixed end beam

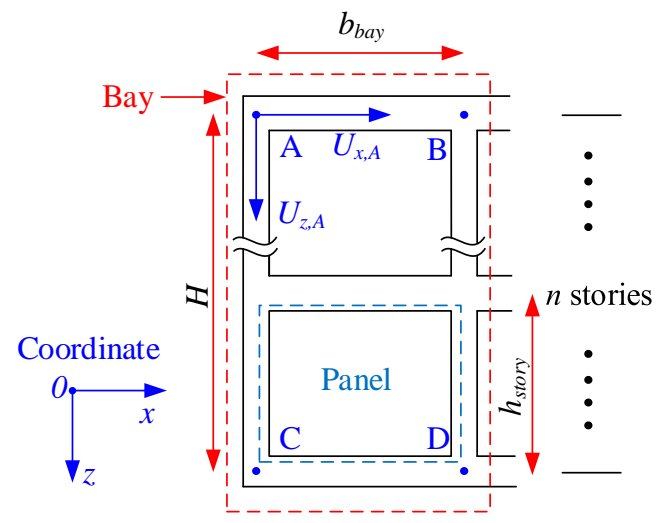

(c) Parameter definition

Fig. 2. (a) and (b) bay distortion modes; (c) parameter definition.

panels, Elkayam and Klar (2019) estimated axial and diagonal strains using the displacements at the four corners of a panel. On the other hand, for framed buildings with infill walls, to account for the beam/slab to column/wall connections, Boone (1996) suggested that the shear strain $\gamma$ could be estimated directly from the maximum slope of the beam/slab with respect to the bay tilt $w$. As illustrated in Figure 2, beams/slabs within the superstructure may undergo either a uniform shear strain ( $\gamma_{1}$ in Figure 2(a)) or a varying profile of shear strain along the beam/slab with a maximum around the mid-point $\left(\gamma_{1}\right.$ in Figure 2(b)). Furthermore, because of ground pressures, the beam/slab shear strains at the foundation level $\left(\gamma_{0}\right)$ can differ from those within the superstructure $\left(\gamma_{1}\right)$.

Importantly, these approaches for estimating average bay (Son and Cording, 2005; Cook, 1994) and local panel (Boone, 1996; Elkayam and Klar, 2019) shear deformations are comparable in terms of tensile strain $\epsilon_{\max }$ and, thus, categories of damage in Table 1. For the considered framed buildings on raft foundations, the high axial stiffness of horizontal elements (slabs) results in $\epsilon_{x x} \approx 0$. Consequently, Equation (2) simplifies to $\epsilon_{\max }=\epsilon_{x z}=\gamma / 2$, which can be computed using (i) $\beta$ from Son and Cording (2005), considering that $\beta \approx \gamma$, (ii) $\gamma$ from Cook (1994) and Elkayam and Klar (2019), or (iii) $\gamma_{0}$ or $\gamma_{1}$ from Boone (1996).

\section{EXPERIMENTAL DESIGN AND PROCEDURE}




\section{Experimental package and building models}

The $4 \mathrm{~m}$ diameter geotechnical centrifuge at the University of Nottingham Centre for Geomechanics (NCG) was used to perform the centrifuge tests. To model the tunneling process, the plane-strain experimental package developed by Zhou (2014) was used, including a strongbox, a transparent acrylic front wall to allow acquisition of digital images of the soil and framed building models, an aluminum back wall, and a flexible membrane model tunnel filled with water (consisting of a rigid aluminum cylinder with enlarged ends sealed within a latex rubber membrane and inserted into the front and back walls of the strongbox). A tunnel volume loss control system was used which included an actuator and water-filled hydraulic cylinder connected by pipes to the water within the model tunnel. The volume loss control system also included a solenoid valve to connect the tunnel, during centrifuge spin-up, to a constant-head standpipe. The standpipe maintained a water pressure within the model tunnel approximately equal to the overburden stress at the depth of the tunnel axis (which increases as the centrifuge spins up) and compensates for any volume change caused by the compression of air within the volume loss control system during centrifuge spin-up. Upon reaching the required g-level, the solenoid valve was configured to connect the tunnel to the actuator/hydraulic cylinder such that water could be extracted from the tunnel, thereby modeling the tunnel volume loss process (full details available in Zhou (2014)). A fine-grained silica sand known as Leighton Buzzard Fraction E was selected for the soil, with minimum $\left(e_{\min }\right)$ and maximum $\left(e_{\max }\right)$ void ratios of 0.65 and 1.01, respectively; all tests were performed with dry soil.

To compare experimental results against comparable tests using equivalent plate model structures (same sand and modeling procedure), the prototype scenario considered by Farrell (2010) and Farrell et al. (2014) was replicated. In Farrell (2010) and in this paper, the following prototype geometry is considered: a tunnel with a diameter $D_{t}$ of $6.1 \mathrm{~m}$, a cover depth $C$ of $8.0 \mathrm{~m}$ (resulting in a cover-to-diameter ratio $C / D=1.3$ ), a building transverse width $B$ of $30 \mathrm{~m}$, and a plane-strain tunnel-soil-structure system. The diameter of the model tunnel used by Farrell (2010) was $D_{t}=82 \mathrm{~mm}$, which differs from $D_{t}=90 \mathrm{~mm}$ in the presented experiments. To match the 
prototype scenario, the tests presented here were carried out at $68 \mathrm{~g}$, instead of $75 \mathrm{~g}$ used by Farrell (2010) (refer to Taylor (1995) for centrifuge scaling laws).

In this study, five framed building models were manufactured by machining and welding aluminum plates and angles. To achieve the plane-strain condition, the model structures extended the full width of the centrifuge strongbox in the direction of the tunnel axis, with continuous vertical walls and horizontal slabs (for both foundations and floors). For the connection between walls and slabs, $60 \%$ of the length along the longitudinal direction was welded (Xu et al., 2019). Considering that the Young's modulus of aluminum and concrete have a similar order of magnitude and that the Young's modulus is not affected by the centrifuge scaling laws (Taylor, 1995), the prototype cross-sectional stiffness of the model slabs and walls replicates reinforced concrete structures in the elastic range of behavior. The building models are $258 \mathrm{~mm}$ long in the tunnel longitudinal direction, leaving a $1 \mathrm{~mm}$ gap at the front/back walls of the $260 \mathrm{~mm}$ wide centrifuge strongbox (the possible effect of this on the accuracy of measured displacement data will be discussed later). The thicknesses $(t)$ of the building elements (foundation, elevated slabs, and walls) for a given building model are the same.

TABLE 2. Framed building configurations.

\begin{tabular}{|c|c|c|c|c|c|c|c|c|c|c|}
\hline \multirow[t]{2}{*}{ Label } & \multirow{2}{*}{$\begin{array}{l}\text { No. of } \\
\text { stories }\end{array}$} & \multirow{2}{*}{$\begin{array}{c}\text { No. of } \\
\text { bays }\end{array}$} & \multicolumn{4}{|c|}{ Model scale (dimension in $\mathrm{mm}$ ) } & \multicolumn{4}{|c|}{ Prototype (dimension in $\mathrm{m}$ ) } \\
\hline & & & $t$ & $H$ & $B$ & $b_{\text {bay }}$ & $t$ & $H$ & $B$ & $b_{\text {bay }}$ \\
\hline F5t5b6L & 5 & 6 & 4.8 & 195.3 & 462.0 & 76.2 & 0.32 & 13.3 & 31.4 & 5.2 \\
\hline $\mathrm{F} 2 \mathrm{t} 5 \mathrm{~b} 6 \mathrm{~L}$ & 2 & 6 & 4.8 & 81.0 & 462.0 & 76.2 & 0.32 & 5.5 & 31.4 & 5.2 \\
\hline $\mathrm{F} 2 \mathrm{t} 3 \mathrm{~b} 6 \mathrm{~L}$ & 2 & 6 & 3.2 & 79.4 & 460.4 & 76.2 & 0.22 & 5.4 & 31.3 & 5.2 \\
\hline $\mathrm{F} 2 \mathrm{t} 3 \mathrm{~b} 3 \mathrm{~L}$ & 2 & 3 & 3.2 & 79.4 & 460.4 & 152.4 & 0.22 & 5.4 & 31.3 & 10.4 \\
\hline $\mathrm{F} 2 \mathrm{t} 3 \mathrm{~b} 3 \mathrm{~S}$ & 2 & 3 & 3.2 & 79.4 & 231.8 & 76.2 & 0.22 & 5.4 & 15.8 & 5.2 \\
\hline
\end{tabular}

Note: $h_{\text {story }}=38.1 \mathrm{~mm}$ at model scale and $2.6 \mathrm{~m}$ at prototype for all frames

Figure 3 and Table 2 show the geometric parameters of the tested frame models. The model frames are labeled based on their structural characteristics as either FxtybzS or FxtybzL: x indicates the number of stories, $y$ the thickness in mm of the structural elements, $\mathrm{z}$ the number of bays, and the suffix $L$ and $S$ are used for transversely long and short structures, respectively. As can be gleaned from Table 2, the number of stories, the thickness $(t)$ of building elements, the building transverse width $(B)$, the number of bays along the transverse width, and the width $\left(b_{b a y}\right)$ of the 
bays varies between building models to isolate the influence of individual structural characteristics on the interaction problem. The height per story $\left(h_{\text {story }}\right)$ is $38.1 \mathrm{~mm}$ for all building models, hence the building height $H$ varies according to the number of stories. A thin layer of sand was bonded to the underside of the building foundations to replicate a rough soil-foundation interface. The GeoPIV digital image analysis technique (White et al., 2003) was used to measure soil and structure displacements. To enhance the performance of the image analysis of the model frames, the front faces of the models were pained matt black and small circular insets were machined within a grid on each face, which were subsequently painted white (see example in Figure S1). A Dalsa Genie Nano-M4020 monochrome camera, with a 12.4-megapixel sensor and $8 \mathrm{~mm}$ Tamron lens, was used in the experiments. Furthermore, single wavelength light-emitting diodes (LEDs) were adopted for the lighting of the model to avoid issues related to chromatic aberration, which has been observed to cause a rainbow effect around the outer areas of images due to refraction of light within the acrylic wall of the strongbox (having a negative impact on the image analysis).

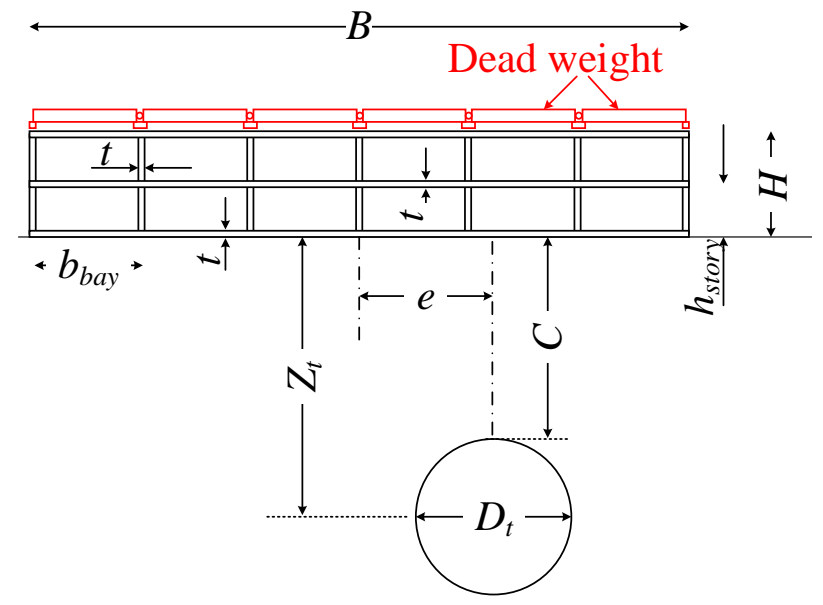

Fig. 3. Experimental layout and parameters.

\section{Test plan and procedure}

A total of 24 centrifuge tests including 2 greenfield (GF) tests and 22 framed buildings tests were performed, as shown in Table 3 and Figure S2. For a given frame model, the relative density of the sand $\left(I_{d}\right)$, the eccentricity $(e)$ of the building with respect to the tunnel, and the building self-weight (SW: standard self-weight; 2SW: doubled self-weight) were varied in the experiments. 
The standard self-weight SW is due to the weight of the aluminum used for the model frames and was calculated for in-flight conditions considering the variation of gravity level along the vertical axis of the frames. The doubled self-weight $2 \mathrm{SW}$ was achieved by adding simply supported (at wall locations) weights to the top of the frame; with this system, the additional weight did not increase the frame stiffness (see Figure S1).

TABLE 3. Centrifuge testing plan.

\begin{tabular}{ccccc}
\hline Total no. tests & Label & $I_{d}(\%)$ & $e / B$ & Weight \\
\hline 2 & Greenfield & $30 \& 90$ & - & - \\
\hline \multirow{2}{*}{16} & \multirow{2}{*}{ F2t3b3L, F2t3b6L, F2t5b6L, F5t5b6L } & 30 & $0 \& 0.2$ & SW \\
& & 90 & 0 & SW\& 2SW \\
\hline \multirow{2}{*}{6} & \multirow{2}{*}{ F2t3b3S } & 30 & $0 \& 0.5$ & SW \\
& & 90 & $0 \& 0.5$ & SW\& 2SW \\
\hline
\end{tabular}

Figure S2 shows the framed building configurations and testing plan. For each of the long frames (label ending in "L"), a total of 4 tests were performed: 2 tests involving a central tunnel $(e / B=0)$ in loose and dense sands $\left(I_{d}=30\right.$ and $\left.90 \%\right)$ for a standard weight SW of the building, 1 test with a central tunnel $(e / B=0)$ in dense sand $\left(I_{d}=90 \%\right)$ and the $2 \mathrm{SW}$ frame, and 1 test for an eccentric tunnel case $(e / B=0.2)$ in loose sand $\left(I_{d}=30 \%\right)$ and SW frame. For the short building model (F2t3b3S), 6 tests were performed; for the loose sand $I_{d}=30 \%$, normalized eccentricity $e / B$ of 0 and 0.5 was tested for the standard building weight SW only, whereas for the dense sand $I_{d}=90 \%$, both normalized eccentricity $(e / B=0 ; 0.5)$ and building weight (SW and $\left.2 \mathrm{SW}\right)$ were varied.

The preparation of dense $\left(I_{d}=90 \%\right)$ and loose $\left(I_{d}=30 \%\right)$ sand models was carried out differently. The dense sand was poured into the container in-line with the model tunnel (consistent with Marshall et al. (2012); Farrell et al. (2014); Franza et al. (2019a)) before moving the package onto the centrifuge cradle. The preparation of loose sand models was carried out with the experimental package on the centrifuge cradle (consistent with Franza and Marshall (2018), who showed good repeatability of results), achieving a relative density of $I_{d}=30 \pm 5 \%$. This relatively quick preparation method for loose soil does imply a greater level of uncertainty relating to soil homogeneity (compared to sand pluviation), however the loose soil test results are mainly used to establish the contrasting effect of loose versus dense soil, hence this issue is not considered to be 
significant. After pouring of the loose sample, the surface was leveled to ensure uniform contact at the soil-foundation interface. In all tunnel-frame interaction tests, the framed building model was carefully placed on the soil surface, the model was gradually spun to $68 \mathrm{~g}$ (at $5 \mathrm{~g}$ the model tunnel was connected to the standpipe), and two stabilization cycles were performed (going from $68 \mathrm{~g}$ to $15 \mathrm{~g}$ and back to $68 \mathrm{~g}$ ); the stabilization cycles are done to help achieve consistency between tests by reducing localized high-stress zones ('hung-up' particles), thereby achieving more uniformly stressed soil profiles. During the centrifuge spin-up, the stress imbalance between the pressures within the model tunnel and the surrounding soil, as well as the increase of the self-weight of the soil and the building, inevitably result in some non-uniformity of stress profiles around the tunnel and an increase of the soil density, however, the effects of these issues have been shown to be minimal (Zhou, 2014; Ritter et al., 2017). After stabilization cycles, tunnel volume loss $V_{l, t}$ was simulated by extracting water from the model tunnel in increments up to a maximum $V_{l, t}$ of $10 \%$. At each $V_{l, t}$ increment, digital images were taken, including both the soil and, when applicable, the model frame structure.

\section{EVALUATION OF BUILDING STIFFNESS}

To evaluate the total stiffness of the long frame models while distinguishing between bending and shear contributions, multiple load-deflection $(P-\delta)$ tests were performed at $1 \mathrm{~g}$ with varying constraint conditions, following the approach of Son and Cording (2007). The frame stiffness was evaluated at $1 \mathrm{~g}$ considering that the model building response (i.e. its stiffness) is not dependent on the centrifuge g-level given the assumption that building elements and node connections remain within the linear elastic range, which was confirmed for this study by checking that $1 \mathrm{~g}$ loading test results agreed for tests done both before and after centrifuge tests. Digital image analysis was used to measure the vertical and horizontal displacements of all wall-slab corners. In particular, simply supported 3-point and 4-point loading tests were carried out with varying spacings between the supports (see Figure 4(a)-(b)). A cantilever loading test was also carried out on model building F2t3b6L with one fixed-end and the free-end subjected to a concentrated load (see Figure 4(c)). The values of experimental bending $\left(E I_{\text {exp }}^{*}\right)$ and shear $\left(G A_{s, \text { exp }}^{*}\right)$ stiffness of the framed building 
models were inferred from the load-deflection tests using Timoshenko beam theory (see formulas in Figure 4). Results are summarized in Table S1, in which tests 1 and 2 refer to 3-point loading tests with a free span length of $B_{1}$ and $B_{2}$, respectively, test 3 is a 4-point loading test with a free span length of $B_{1}$, and test 4 is the cantilever test with a free deflection length of $B_{3}$.

The values of experimental bending and shear stiffness $\left(E I_{\text {exp }}^{*}\right.$ and $\left.G A_{s, \exp }^{*}\right)$ were evaluated for the long frames F2t3b6L, F2t5b6L and F5t5b6L by combining results from two of the loaddeflection tests (i.e. two unknowns in two equations). Interestingly, the average experimental building bending stiffness, $E I_{\text {exp,avg }}^{*}$, was found to be about one-third of the theoretical bending stiffness obtained using parallel axis theory, $E I_{\text {parallel }}^{*}$. Only a 4-point load-deflection test was performed for the long frame F2t3b3L because, for this frame, there is no central column. To calculate the shear stiffness of this frame from the single test, it was assumed that $E I_{\text {exp }}^{*}$ of F2t $3 \mathrm{~b} 3 \mathrm{~L}$ was equal to the average $E I_{\text {exp }}^{*}$ of $\mathrm{F} 2 \mathrm{t} 3 \mathrm{~b} 6 \mathrm{~L}$, which is reasonable given the identical element thickness and transverse width. No load-deflection tests were performed for the short frame; it was assumed that the values of $E I_{\text {exp }}^{*}$ and $G A_{s, \text { exp }}^{*}$ for the short frame matched those of the long frame F2t3b6L because of the identical element thickness and bay width.

(a) 3-point test
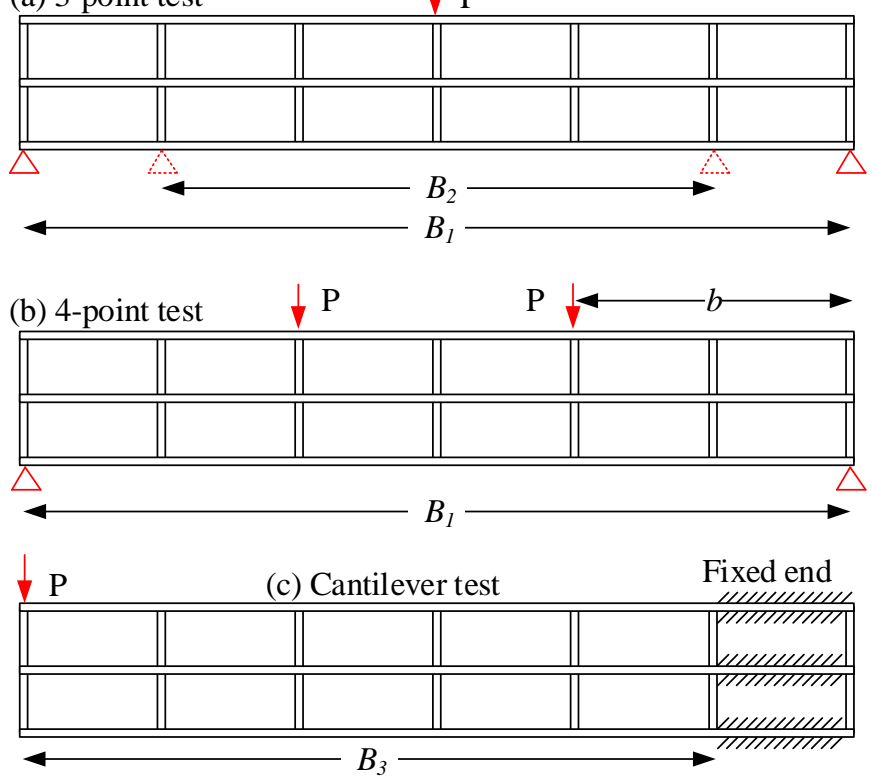

Timoshenko Beam Theory

(a) 3-point loading test:

$$
\begin{aligned}
& \delta_{\text {total }}=\delta_{\text {shear }}+\delta_{\text {bending }}=\frac{P B}{4 G A_{s}}+\frac{P B^{3}}{48 E I} \\
& \frac{\delta_{\text {shear }}}{\delta_{\text {bending }}}=\frac{12 E I}{B^{2} G A_{s}}=12 F
\end{aligned}
$$

(b) 4-point loading test:

$$
\begin{aligned}
& \delta_{\text {total }}=\delta_{\text {shear }}+\delta_{\text {bending }}=\frac{P b}{G A_{s}}+\frac{P l\left(3 B^{2}-4 b^{2}\right)}{24 E I} \\
& \frac{\delta_{\text {shear }}}{\delta_{\text {bending }}}=\frac{24 E I}{\left(3 B^{2}-4 b^{2}\right) G A_{s}}=\frac{24 B^{2}}{\left(3 B^{2}-4 b^{2}\right)} F
\end{aligned}
$$

(c) Cantilever beam test:

$$
\begin{aligned}
& \delta_{\text {total }}=\delta_{\text {shear }}+\delta_{\text {bending }}=\frac{P B}{G A_{s}}+\frac{P B^{3}}{3 E I} \\
& \frac{\delta_{\text {shear }}}{\delta_{\text {bending }}}=\frac{3 E I}{B^{2} G A_{s}}=3 F
\end{aligned}
$$

Fig. 4. Configuration of experimental tests to evaluate building stiffness.

Finally, Table S1 also reports the value of $\delta_{\text {shear }} / \delta_{\text {bending }}$ in the 3-point tests with a free span 
length of $B_{1}$. Interestingly, shear deflection dominates the total deformation for all the frames, with a shear deflection ranging between about 8 and 50 times the bending deflection. This is also confirmed by the measured deformed shape of frame F2t3b6L in Figure 5. The axial strains within the top and bottom slabs were minimal (indicating minimal global bending deformation of the structure), whereas differential settlements between adjacent bays occurred due to the shear deformation of panels.

Furthermore, as displayed by Figure 5(a), the deflection in a shear-dominated building is the result of the changing of the sign of the shear deformations, rather than the absolute value of shear distortions. Figure 5(b) also illustrates that (neglecting the presence of the fixed bay), an approximately straight settlement profile (associated with $D R \approx 0$ and a given total tilt $\omega^{\prime}$ ) may be associated with large shear deformations if local tilt $w$ is prevented (e.g. $\beta=S$ when $w=0$ ). On the other hand, for a frame that is able to undergo tilt $w$, a straight settlement profile can indicate rigid body motion with no deformations (e.g. $\beta=0$ when $S=w$ ).
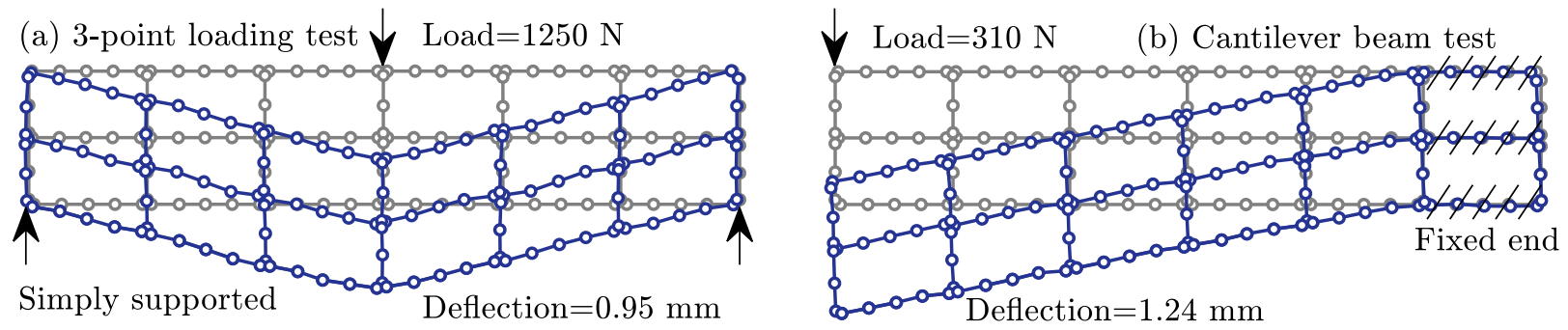

Fig. 5. Deformed shape of F2t3b6L in the load-deflection tests (scale factor: 50).

To compare these frame results against the centrifuge data for plates from Farrell et al. (2014) (labelled as 'STR'), "pure" equivalent bending stiffness, $E I_{E B, e q}^{*}$, of building models were computed assuming rigid behavior in shear (i.e. $G A_{s}$ is infinite and pure bending deformations occur, as for an Euler-Bernoulli beam). The thicknesses of the corresponding equivalent plates $t_{e q}$ and $\log _{10}\left(E I_{E B, e q}^{*}\right)$ are reported in Table 4 in prototype scale; the latter is provided because tunnelstructure interaction varies with relative stiffness parameters according to a semi-log scale (Potts and Addenbrooke, 1997; Mair, 2013).

From Table 4, it can be seen that the stiff frame F5t5b6L and the equivalent plate STR4 have 
TABLE 4. Equivalent $E I$ of structures and pressure beneath the foundations (prototype scale).

\begin{tabular}{llccccl}
\hline Structure & $\begin{array}{l}E I_{E B, e q}^{*} \\
(\mathrm{GNm})\end{array}$ & $\begin{array}{c}t_{\text {eq }} \\
(\mathrm{m})\end{array}$ & $\begin{array}{c}\log _{10}\left(E I_{E B, \text { eq }}^{*}\right) \\
(\mathrm{Nm})\end{array}$ & $\begin{array}{c}E I_{\text {exp,avg }}^{*} \\
(\mathrm{GNm})\end{array}$ & $\begin{array}{c}G A_{\text {s,exp,avg }}^{*} \\
(\mathrm{MNm})\end{array}$ & $\begin{array}{l}\text { Pressure }(\mathrm{kPa}) \\
\mathrm{SW} / 2 \mathrm{SW}\end{array}$ \\
\hline F2t3b3L & 1.48 & 0.63 & 9.17 & - & 14 & $19.4 / 38.8$ \\
F2t3b6L & 3.24 & 0.82 & 9.51 & 60 & 42 & $22.8 / 45.6$ \\
F2t5b6L & 9.31 & 1.17 & 9.97 & 88 & 130 & $34.8 / 69.6$ \\
F5t5b6L & 21.11 & 1.54 & 10.32 & 970 & 270 & $73.5 / 147$ \\
STR2 & 0.32 & 0.38 & 8.50 & - & - & $9.9 /$ \\
STR3 & 2.46 & 0.75 & 9.40 & - & - & $19.8 /$ \\
STR4 & 19.69 & 1.50 & 10.30 & - & - & $39.7 /$ \\
\hline
\end{tabular}

Note: Pressure for SW and 2SW of F2t3b3S are 23.9 and $47.8 \mathrm{kPa}$, respectively

nearly identical values of $E I_{E B, e q}^{*}$; however, the average pressure beneath model F5t5b6L is almost twice that of STR4. Frame F2t3b6L has slightly larger values of $E I_{E B, e q}^{*}$ and pressure than plate STR3. The rather flexible plate STR2 is less stiff than the frame F2t3b3L. Finally, note that the variation of $\log _{10}\left(E I_{E B, e q}^{*}\right)$ values for all frames is rather limited. The context of these results will be clearer in later sections of the paper where results from tunnel-building interaction tests are compared.

\section{CENTRIFUGE RESULTS}

\section{Framed building foundation displacements and comparison with equivalent plates}

The settlements, $U_{z}$, of the foundations of the model framed buildings and equivalent plates at a tunnel volume loss $V_{l, t}=1 \%$ are presented in Figure 6 along with the greenfield settlements at the surface. The greenfield (GF) settlements between the two data sets (GF-Xu is the new data in Figure 6(a); GF-Farrell in Figure 6(b) is from Farrell (2010)) are sufficiently consistent to allow comparison of the tunnel-structure interaction outcomes. It was found that (not reported here), after about $V_{l, t}=3 \%$, differences between the greenfield data became greater, hence comparisons are not made outside of this range of $V_{l, t}$.

In terms of minimum and maximum structure settlements, frames and plates follow the same principle: 1) the lower the total stiffness (depending on $t_{e q}$ ) of the structure, the greater the maximum settlement; and 2) the stiffer the structure, the greater the minimum settlement. However, the selfweight (discussed later) also has a role. Both the frames and plates responded rigidly to tunneling in the horizontal direction, resulting in very small differential horizontal displacements in the frame 

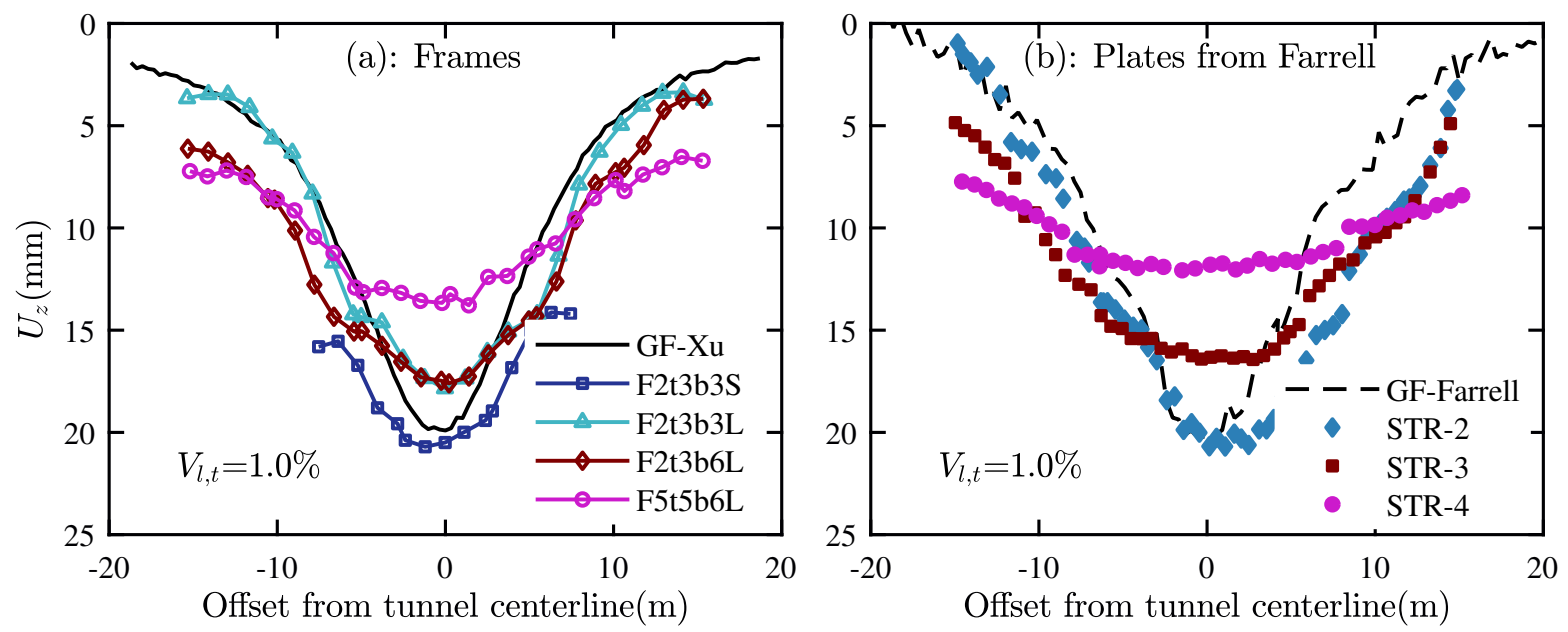

Fig. 6. Vertical displacement of model frames and equivalent plates for $e / B=0$ and $V_{l, t}=1 \%$.

foundation slabs and plates, as reported later.

Figure 6 indicates that all frames have both sagging and hogging regions, whereas the plates mostly undergo a sagging deformation mode, regardless of their stiffness and transverse width. On the other hand, for the structures with similar equivalent bending stiffness, $E I_{E B, e q}^{*}$ (refer to Table 4), framed buildings tend to have a more flexible response to tunneling than equivalent plates (compare frame F5t5b6L against plate STR-4, and frame F2t3b6L against plate STR-3). This point can also be demonstrated by comparing frame F2t3b3L and plate STR-2, in which the frame not only has a large deflection ratio in the sagging zone, but also displays significant hogging zones. This mechanism is likely due to the role of the shear flexibility being negligible $\left(1 / G A_{s} \approx 0\right)$ for the equivalent isotropic plates. Similar phenomena for frames have also been observed in numerical studies (e.g. Boldini et al. (2018)), whereas Franza et al. (2019b) discussed how equivalent EulerBernoulli beams and structures that are rigid in shear tend to undergo singular sagging or hogging settlement profiles for central and eccentric tunnels, respectively.

These observations indicate that, although frames F5t5b6L and F2t3b6L have similar values of $E I_{E B, e q}$ as plates STR-4 and STR-3, respectively, the structural characteristics (i.e. those producing a shear-dominated or a bending-dominated deformation behavior) have a significant effect on the resulting soil-structure interactions. The presented data therefore confirms that the tunneling-induced settlement profile depends on the structure configuration; furthermore, the use 
of equivalent pure bending $E I_{E B, e q}$ representations of building behavior may lead to significant errors for some tunnel-building interaction cases.

\section{Effect of building weight}

To evaluate the impact of structure weight, the vertical $\left(U_{z}\right)$ and horizontal $\left(U_{x}\right)$ displacements of the foundations of the model framed buildings and soil beneath the foundations are presented in Figure 7 at $V_{l, t}=2 \%$ for a selection of centrifuge tests, together with greenfield test results. Results are compared using tunnel volume loss $V_{l, t}$ rather than soil volume loss $V_{l, s}$ (calculated as the integration of surface settlements) because $V_{l, t}$ is a more generally used parameter that relates to the performance of tunneling operations. In Figure 7, markers are used for foundation displacements, and lines are used for surface soil displacements in greenfield and framed building tests. The data can be used to distinguish a gap forming between the soil and foundation in some tests; to better illustrate this feature, the maximum gap height is plotted against tunnel volume loss $V_{l, t}$ in Figure S3 of the Supplemental Data. In some cases, the data indicate that the foundations settled slightly more than the soil beneath them at the edges of the building models. This inconsistency occurs because the front of the frames was not immediately against the inner face of the acrylic wall (also observed in Farrell (2010) and Ritter et al. (2017)). As a result, a negative gap height in Figure S3 relates to measurement errors.

First, settlement profiles in Figure 7 are considered. The dense sand data (dark color) in Figure 7 can be used to demonstrate the effect of building weight: an increase of weight increases the distortions of centrally located structures (see Figure 7(a), (c)-(f)), and decreases the size of the gap formed between the soil and foundation, whereas the effect of weight on settlement at the edges of the buildings is minor. Note that, at $V_{l, t}=2 \%$, a gap was not observed beneath the centrally located structures with double weight $2 \mathrm{SW}$, except for frame F2t5b6L, whereas only frame F2t3b3L did not show a gap for standard weight SW. Importantly, for the short frame F2t3b3S, the influence of building weight was lower for the eccentric case $e / B=0.5$ than for the central case $e / B=0$.

An interesting comparison can also be made between tests with frame F2t5b6L with $2 \mathrm{SW}$ and frame F5t5b6L with SW because they have a similar pressure acting beneath the foundations, but 
Greenfield

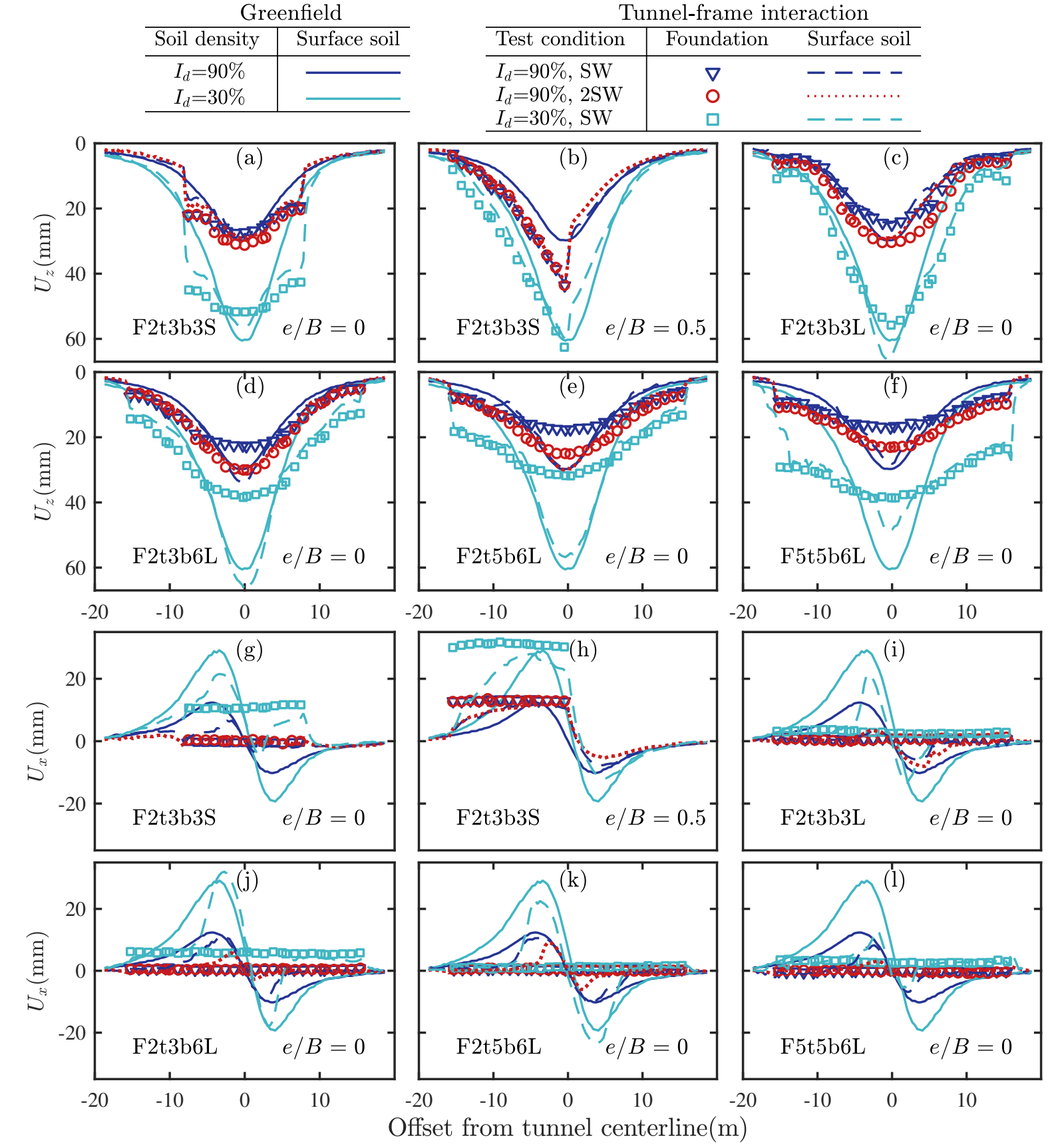

Tunnel-frame interaction

Fig. 7. Displacement of model frame foundations and underlying soil at $V_{l, t}=2 \%$.

different stiffness (compare dark circles in Figure 7(e) with dark triangles in Figure 7(f)). As expected, a larger deflection ratio and smaller gap (quantified in Figure S3(a)) are observed in the former test since the frame has a smaller stiffness.

In Figure 7(g)-(1), all frames with $e / B=0$ experienced negligible horizontal displacements 
(except for the short frame in Figure 7(g) on loose soil) and the restriction provided by the foundation roughness and building weight acted to reduce horizontal soil displacements. When the building weight was doubled (i.e. $2 \mathrm{SW}$ compared to $\mathrm{SW}$ ), the restraint provided by the foundations to the underlying soil was increased and horizontal soil displacements decreased. In the tests where nearly no gap was observed, surface horizontal soil displacements were found to be very small or negligible (e.g. test 2SW in Figure 7(j)-(1)).

\section{Effect of soil relative density}

First, the effect of soil relative density is discussed using the data in Figure 7 for a constant tunnel volume loss $V_{l, t}=2 \%$. Because of the different volumetric response of the dense and loose sands to tunneling induced soil shear strains, the greenfield surface settlements in the loose sand are much higher than in the dense sand (also refer to the tunnel versus soil volume loss data in Figure 9). This caused the frames to experience larger average and differential settlements in the loose sand tests compared to the dense sand. Relative density also affected the size of gap that formed at the soil-foundation interface; for example see Figure S3 for $V_{l, t}=2 \%$, where larger gaps formed for $I_{d}=30 \%$ because of the higher levels of ground movement near the surface.

To evaluate the soil-structure interaction for settlement troughs of equal volume, results are also compared for a given soil volume loss $V_{l, s}=1.6 \%$. First, trough characteristics of the greenfield settlements for $I_{d}=30$ and $90 \%$ are listed in Table 5, which were obtained by fitting modified Gaussian curves (Vorster et al., 2005) to the settlement data. The settlement trough for loose soil is slightly wider (a larger $i$ and $K$ ) than in dense sand, although the maximum settlement values are close. The wider greenfield settlement curve for $I_{d}=30 \%$ should result in lower differential settlements and distortion levels of a building than the dense soil $I_{d}=90 \%$. The settlements of central building foundations $(e / B=0)$ with standard weight $(\mathrm{SW})$ are presented in Figure 8 for $V_{l, s}=1.6 \%$ (calculated using soil settlements beneath the building foundations). Except for the most flexible frame F2t3b3L, a greater maximum settlement is observed for the building foundations in loose soil. Differential settlements along corresponding building foundations are qualitatively similar for the given magnitude of soil volume losses; a more direct assessment of 
building deformations is given later by considering angular distortions of the buildings.

TABLE 5. Greenfield settlement trough characteristics (prototype scale).

\begin{tabular}{cccccc}
\hline$I_{d}(\%)$ & $V_{l, t}(\%)$ & $V_{l, s}(\%)$ & $U_{z, \max }(\mathrm{mm})$ & $i(\mathrm{~m})$ & $K$ \\
\hline 30 & 1 & 1.6 & 31.68 & 3.79 & 0.344 \\
90 & 2.5 & 1.6 & 32.78 & 3.32 & 0.302 \\
\hline
\end{tabular}
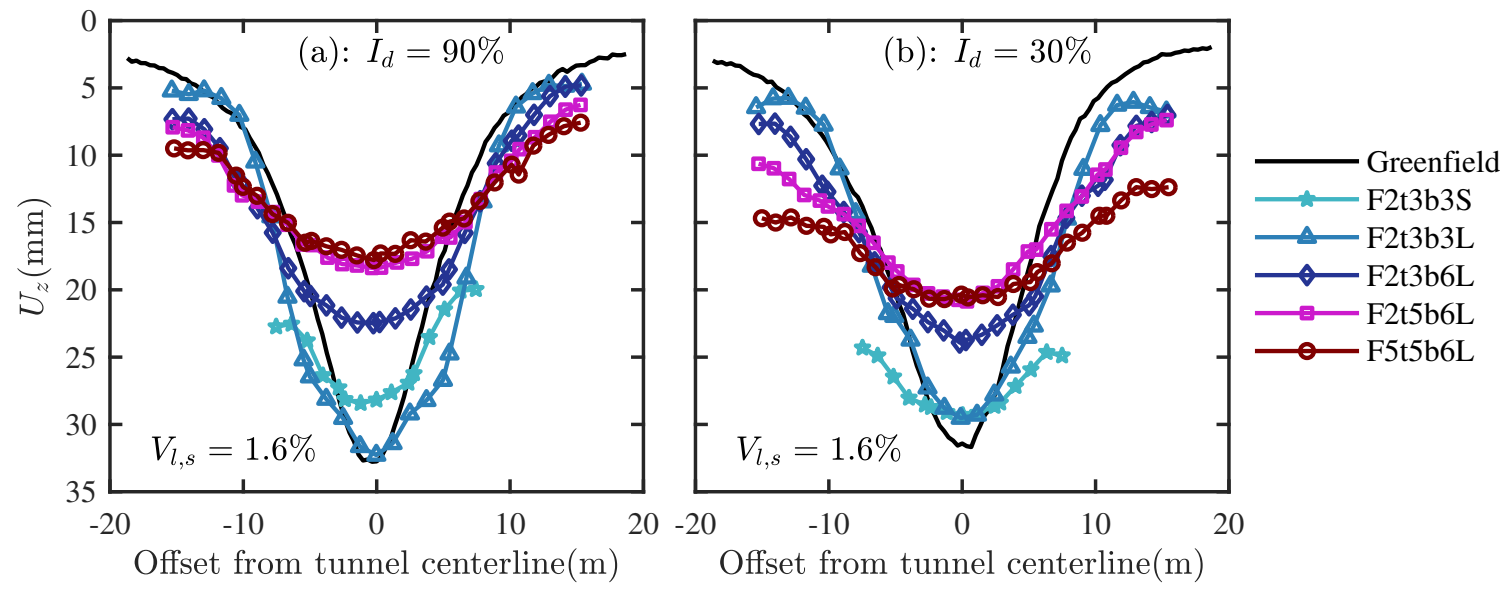

Fig. 8. Displacements of selected model foundations $(e / B=0, \mathrm{SW})$ at identical $V_{l, s}=1.6 \%$ : (a) $I_{d}=90 \%$ and (b) $I_{d}=30 \%$.

\section{Effect of relative tunnel-building location}

The data in Figure 7(a) and (b) can be compared to study the effect of relative tunnel-building location. Results indicate that the eccentric short frames in (b) have a smaller relative deflection $\Delta$ than the central frames in (a) because of building tilt $w$ for the eccentric cases. This effect of $e / B$ on relative deflections was not as significant for the long frames (not presented here), partly because the maximum eccentricity $e / B$ achievable in the centrifuge tests for the long frames was 0.2 , compared to 0.5 for the short frames.

At the soil-structure interface, the increase of building eccentricity $e / B$ reduced the size of the gap formed beneath the foundation (see also Figure S3(b)), in agreement with the trends highlighted by Franza and DeJong (2019). The short frames with normalized eccentricity $e / B=0.5$ show horizontal movements that are approximately equal to the maximum greenfield horizontal displacement (see Figure 7(h)), whereas for the eccentric long frames with $e / B=0.2$ (not presented here), horizontal movements of the foundations were minimal. The horizontal displacements of the soil beneath the short frame with $e / B=0.5$ are generally greater than greenfield ground 
displacements; this is due to the compliance of the soil-foundation interface (see Figure 7(h)).

\section{Soil volume losses}

It could be argued that the variation of soil volume loss $V_{l, s}$ at the surface (given by the integration of the surface settlements) with building self-weight may impact the structure distortion. To investigate this aspect, soil volume losses $V_{l, s}$ against tunnel volume loss $V_{l, t}$ is shown in Figure 9. Interestingly, there is only a minor increase in the value of $V_{l, s}$ when the self-weight of the frame is doubled for central tunnels in dense sand, while an increase of normalized eccentricity $e / B$ for loose sand slightly reduced $V_{l, s}$. Soil relative density $I_{d}$ clearly dominated the $V_{l, s}-V_{l, t}$ relationship for all considered structures, which impacted the overall structure settlement level.
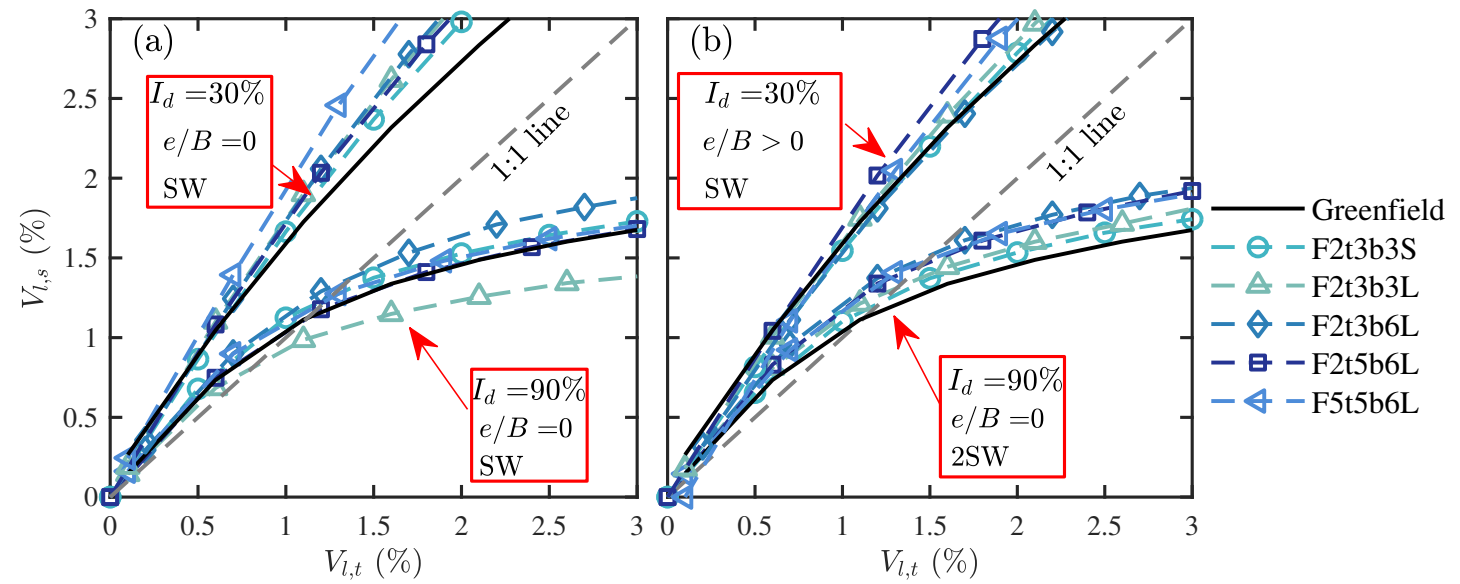

Fig. 9. Variation of soil volume loss with tunnel volume loss.

\section{Deformation parameters and modification factor approach}

Deflection ratios, $D R$, of the framed buildings in selected tests are presented in Figure 10(a) and (b) according to normalized eccentricity for tunnel volume loss up to 3\%. A complete set of results of building deformation parameters $\left(D R, M^{D R}\right.$ and $\left.\beta\right)$ are reported in Figure S4. To compute the deflection ratios of the buildings, both the length of sagging and hogging zones as well as the relative deflections were determined from the frame settlement profiles (considering points at raft-column intersections and the centre of rafts between two columns). On the other hand, greenfield values of $D R$ and lengths of sagging/hogging zones were interpolated using modified Gaussian curves. 

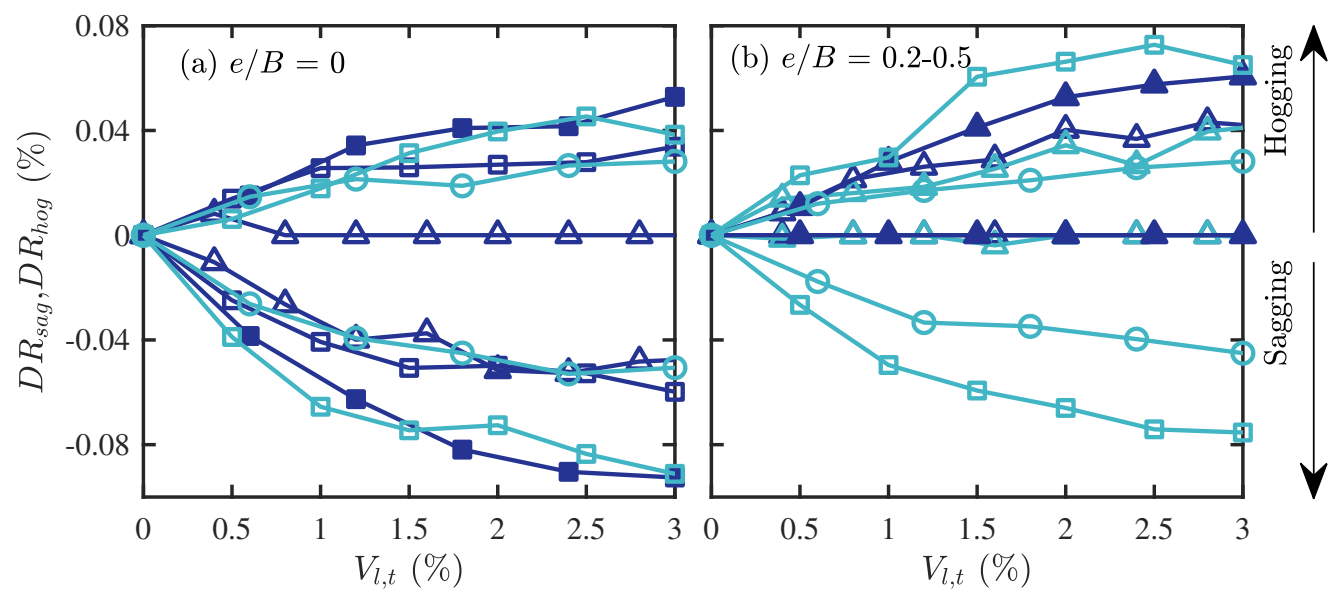

Marker fill

$\Delta$ SW

$\Delta 2 \mathrm{SW}$

Marker type

$\Delta$ F2t3b3S

口 F $2 \mathrm{t} 3 \mathrm{~b} 6 \mathrm{~L}$

O F $2 \mathrm{t} 5 \mathrm{~b} 6 \mathrm{~L}$

Color type

$\triangle I_{d}=30 \%$

$\Delta I_{d}=90 \%$

Fig. 10. Building deflection ratios against tunnel volume loss in selected tests.

Results of $D R$ in Figure 10 show that long frames underwent both sagging and hogging deformations, whereas short frames mostly displayed either a sagging or hogging deformation mode when $e / B=0$ or 0.5 , respectively. For the centrally located long frames, sagging $D R$ values tend to be larger in magnitude than hogging values. For all tests with long framed buildings, a stiffer frame tends to reduce $D R$ in both sagging and hogging. Results in Figure 10 also show that the increase of building weight (compare solid and hollow markers) tends to increase $D R$ values, especially in sagging. In addition, the decrease of soil relative density (from $90 \%$ to $30 \%$; compare results in dark and light colors) increases $D R$ values for a given $V_{l, t}$; this effect is significant for the very flexible frame F2t3b3L (displayed in Figure S4(b)-(c)) because the frame tends to conform to the tunneling-induced ground movements. Finally, results in Figure 10(a)-(b) indicate that the increase of building eccentricity has a reducing effect on $D R$ values for most frames.

Deflection ratio modification factors, $M^{D R}$, of the framed buildings in selected tests are presented in Figure 11 according to normalized eccentricity for tunnel volume loss up to 3\%. Results of the deflection ratio modification factors $M^{D R}$ in Figure S4 show that most $M^{D R}$ trends are characterized by a steady decrease with $V_{l, t}$ over the investigated range, and a greater rate of decrease is obtained for the loose soil. The decrease of $M^{D R}$ with $V_{l, t}$ is due to both soil stiffness degradation that occurs with tunnel volume loss (Farrell et al., 2014) and, when present, gap formation (quantified in Figure S3) (Deck and Singh, 2012; Franza and DeJong, 2019). Furthermore, increased building stiffness tends to decrease $M^{D R}$. In the sagging deformation mode, the increase of soil 

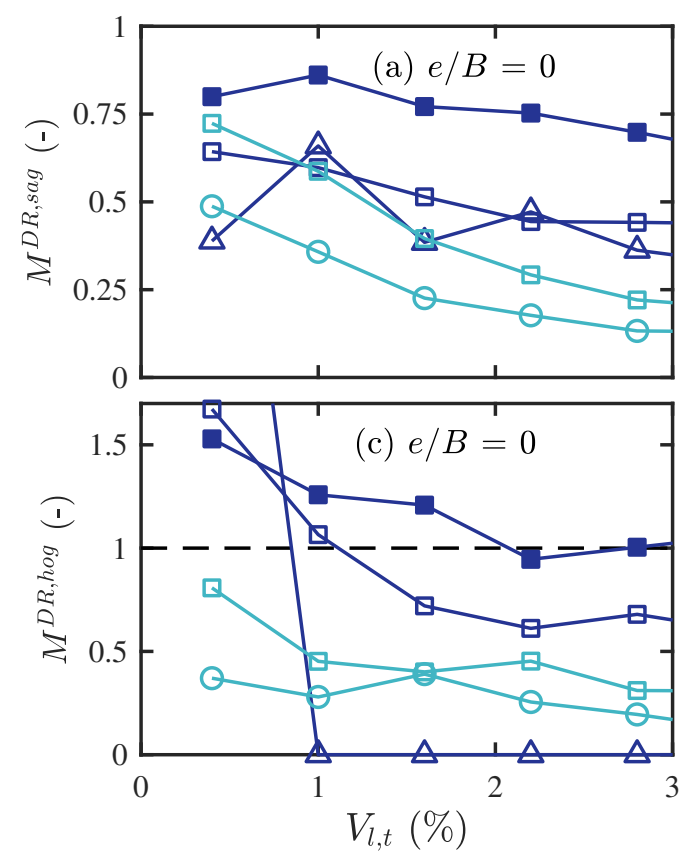
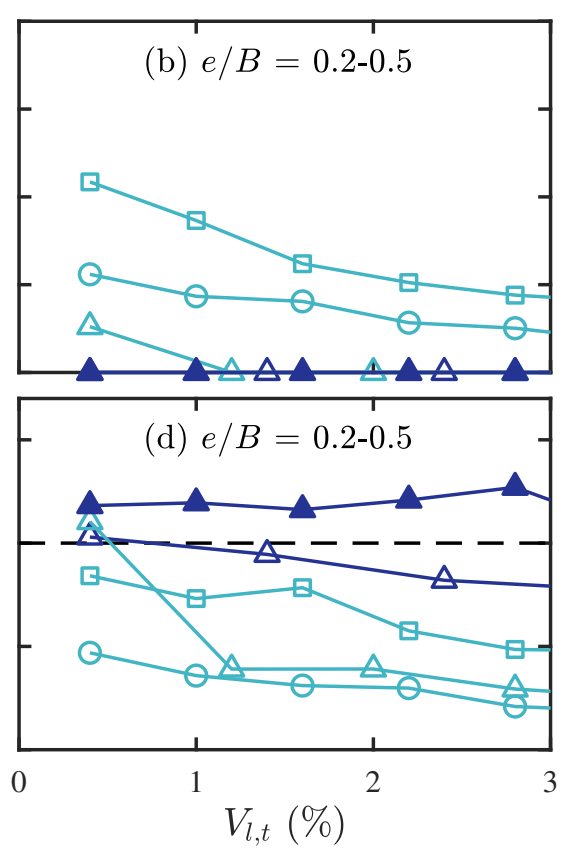

Marker fill

$\triangle \mathrm{SW}$

$\Delta 2 \mathrm{SW}$

Marker type

$\triangle$ F2t3b3S

口 $\mathrm{F} 2 \mathrm{t} 3 \mathrm{~b} 6 \mathrm{~L}$

O F $2 \mathrm{t} 5 \mathrm{~b} 6 \mathrm{~L}$

Color type

$\triangle I_{d}=30 \%$

$\triangle I_{d}=90 \%$

Fig. 11. Building modification factors of deflection ratios against tunnel volume loss in selected tests.

relative density (from 30\% to 90\%), building weight, and normalized eccentricity tend to increase, increase, and decrease, respectively, the $M^{D R, s a g}$ values for a given $V_{l, t}$. The values of $M^{D R, h o g}$ tend to be larger than $M^{D R, s a g}$ for all tests (see Figure S4) due to 1) the effect of gap formation in the sagging zone, and 2) embedment at the building corners in the hogging zones. Generally, $M^{D R, h o g}$ values in the dense soil tests tend to be larger than in the loose soil tests. Interestingly, the increase of frame eccentricity tends to increase $M^{D R, h o g}$, likely because a larger portion of the building was subjected to the hogging deformation mode in these cases.
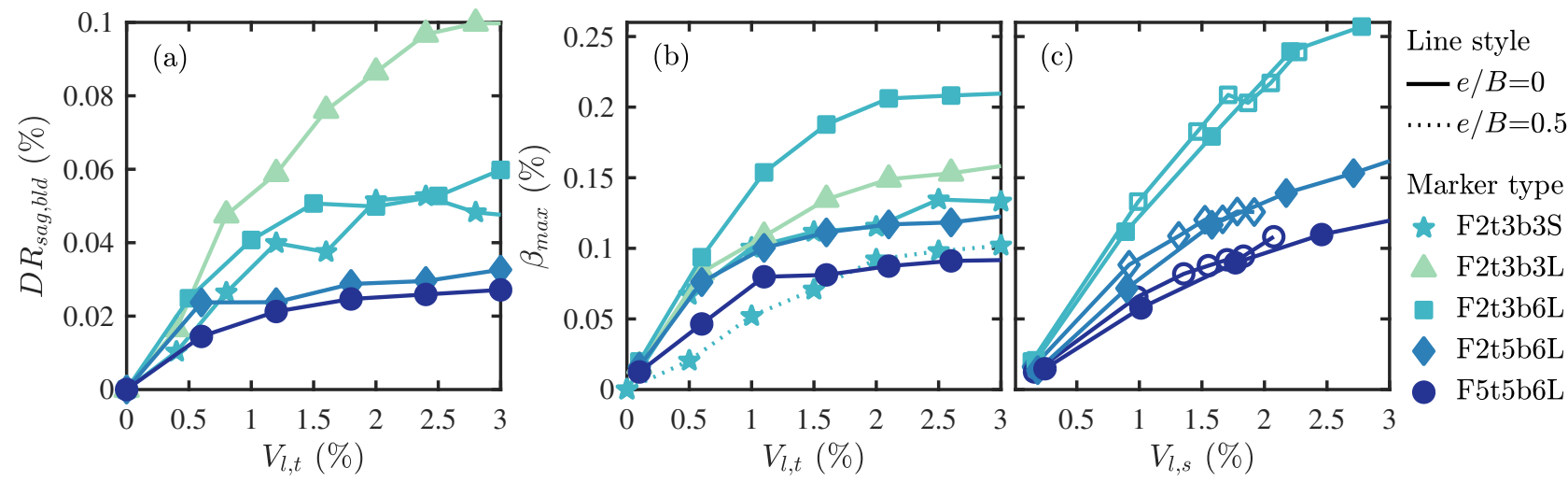

Fig. 12. Building deflection ratios and angular distortions against tunnel/soil volume loss in selected tests $\left(I_{d}=90 \%\right.$ and $\left.\mathrm{SW}\right)$. 
The maximum values of the building angular distortion, $\beta$, within bays in selected tests are reported against $V_{l, t}$ in Figure 12(b), along with the maximum magnitude of the building deflection ratios in sagging, $D R_{\text {sag,bld }}$, in Figure 12(a). It is found that the effects of building weight, soil relative density, and building eccentricity on $\beta$ mostly agree (qualitatively) with the trends relating to $D R$ (see figures $\mathrm{S} 4$ and 10$)$.

Despite this agreement, building angular distortion is not only related to building stiffness, but also to the structural configuration. On one hand, for the long frames F2t3b6L, F2t5b6L, and F5t5b6L with the same bay and building widths, the increase of building stiffness (due to thicker elements or additional stories) decreased the angular distortion (see Figure 12(b)). On the other hand, shorter bay length is observed to increase bay distortions; in Figure 12(b), the most flexible frame F2t3b3L (with a long bay length; consider stiffness values in Table 4) has $\beta$ values lower than F2t3b6L (with similar characteristics but shorter bays), although F2t3b3L did undergo the largest $D R$ values. Furthermore, Figure 12(a) also displays that the short frame F2t3b3S and the long frame F2t3b6L have similar sagging deflection ratios, however the angular distortion for the shorter frame is much smaller. These results indicate that the deflection ratios are not always as efficient as angular/shear distortions for quantifying framed building distortions.

It is also of interest to analyze building angular distortion $\beta$ for a given soil volume loss $V_{l, s}$. In Figure 12(c), the maximum $\beta$ for tests with $e / B=0$ and SW (see Figure 8) are plotted against $V_{l, s}$ (full data set is in Figure S5). Interestingly, for a given $V_{l, s}$, Figure 12(c) shows that there is little difference in building deformation for loose and dense sand.

Finally, the role of structure tilt on the frame deformations is discussed. The short frame F2t3b3S with $e / B=0.5$ can be regarded as half of the long frame $\mathrm{F} 2 \mathrm{t} 3 \mathrm{~b} 6 \mathrm{~L}$ with $e / B=0$. Interestingly, the eccentric short frame F2t3b3S has a much smaller angular distortion than the central long frame F2t3b6L in Figure 12(b), despite the fact that they have similar values of $D R_{h o g}$ and $M^{D R, h o g}$ (see Figure 10 and Figure 11(c)-(d)). As will be displayed later by plotting the frame deformed shapes, this behavior is due to the fact that the eccentric (short) frame can tilt to accommodate ground settlements, whereas tilt is prevented by the symmetry condition for the central structure. These 
outcomes confirm that $D R$ alone does not always provide an accurate description of distortion for a shear-dominated building.

\section{Level of damage based on strain theory}

Figure 13 shows frame deformed shapes and ground movements for 14 of the centrifuge tests at $V_{l, t}=2 \%$; for F5t5b6L only the results of the first and second stories are presented. To quantify the deformation levels of bays and panels across the frames, indicators (markers) are used which are associated with a range of shear strains $\gamma$ and a category of damage; the category was computed using a Mohr's circle of strain under plane-strain conditions (i.e. $\epsilon_{\max }=\epsilon_{x z}$ for framed buildings on raft foundations). In Figure 13, the left and right columns relate to dense and loose sands, respectively. Indicators plotted within bays are indicative of $\gamma$ values (calculated based on the displacements at the corners of bays), whereas indicators of $\gamma_{0}$ and $\gamma_{1}$ (computed from slab slopes) are placed along the foundations or floor slabs. A color scheme was adopted to denote low (category of $0-1$ ); medium (category 2), and high (category 3+) levels of deformation (see Table 1). For reference, the values of $\gamma$ presented in Figure 13 are also listed in Table S2 of the Supplemental Data.

First, the deformed shapes of the short frame F2t3b3S tests are considered to evaluate the effects of normalized eccentricity $e / B$ and soil relative density $I_{d}$. Figure 13(a) and (b) show the results for frame $\mathrm{F} 2 \mathrm{t} 3 \mathrm{~b} 3 \mathrm{~S}$ with $e / B=0.5$. Although the frame tested in loose sand was subjected to larger settlements and global tilt, the same level of deformation is observed for both soil densities: low levels in all bays and medium levels at the foundation level (due to the ground pressure). When the same short frame was centrally located with $e / B=0$ (Figure 13(c) and (d)), the external bays underwent larger distortions (medium level for all slabs according to Boone (1996)) than for the eccentric configuration $e / B=0.5$ in Figure 13(a) and (b). For $e / B=0$, the looser soil led to slightly increased values of $\gamma$ related to panel deformations.

The impact of the transverse width $B$ is now considered. First, the short structure F2t3b3S can be seen as a portion (sub-structure) of the long frame F2t3b6L. Comparing Figure 13(c) to (g) and Figure 13(d) to (h) shows that increasing building transverse width (from spanning only the 


$\begin{array}{cclll} & \gamma(\%) & \text { Category } & & \text { Marker type } \\ \square I_{d}=90 \% & 0.3 & 3+ & V_{l, t}=2 \% & \\ \square I_{d}=30 \% & 0.15 & 2 & & \text { Boone }\left(\gamma_{0}, \gamma_{1}\right) \\ & 0 & 0-1 & \operatorname{Cook}(\gamma)\end{array}$

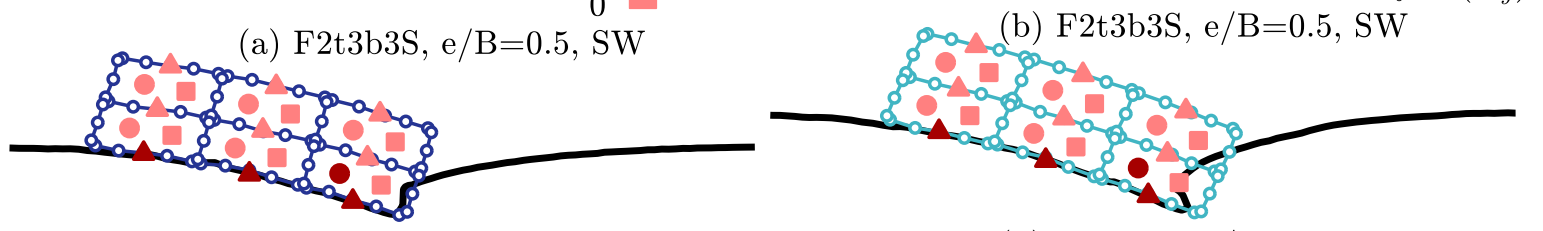

(c) $\mathrm{F} 2 \mathrm{t} 3 \mathrm{~b} 3 \mathrm{~S}, \mathrm{e} / \mathrm{B}=0, \mathrm{SW}$

(d) F2t3b3S, e/B=0, SW
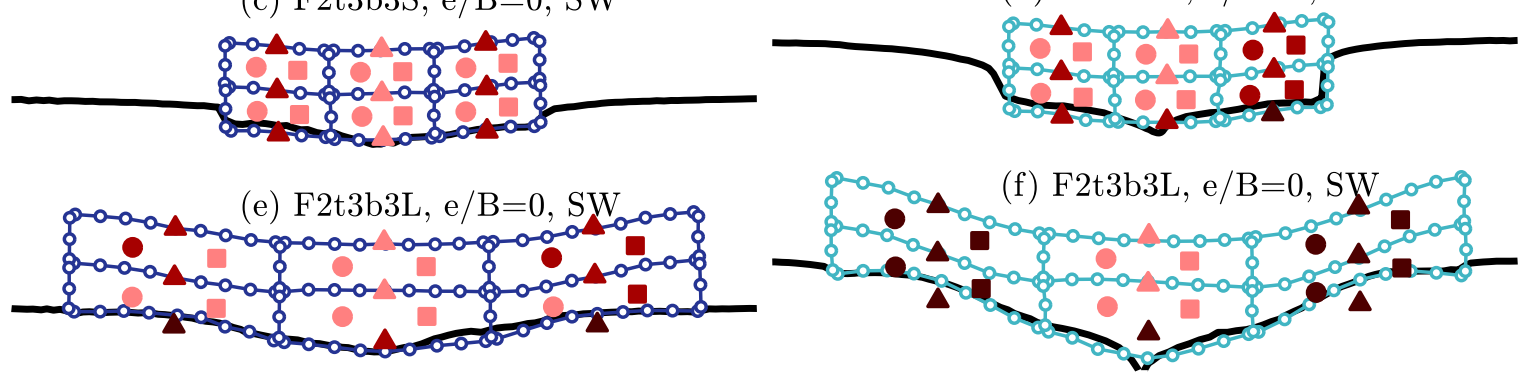

(g) $\mathrm{F} 2 \mathrm{t} 3 \mathrm{~b} 6 \mathrm{~L}, \mathrm{e} / \mathrm{B}=0$, SW

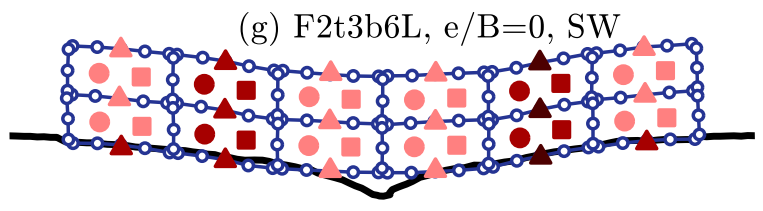

(h) F2t3b6L, e/B=0, SW

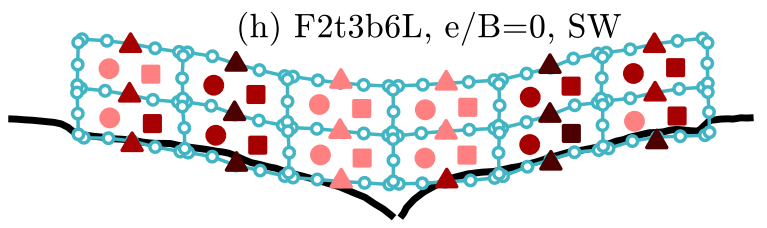

(i) $\mathrm{F} 2 \mathrm{t} 3 \mathrm{~b} 6 \mathrm{~L}, \mathrm{e} / \mathrm{B}=0,2 \mathrm{SW}$

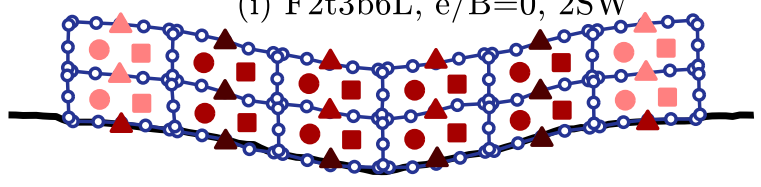

(j) $\mathrm{F} 2 \mathrm{t} 3 \mathrm{~b} 6 \mathrm{~L}, \mathrm{e} / \mathrm{B}=0.2, \mathrm{SW}$

(k) $\mathrm{F} 2 \mathrm{t} 5 \mathrm{~b} 6 \mathrm{~L}, \mathrm{e} / \mathrm{B}=0,2 \mathrm{SW}$
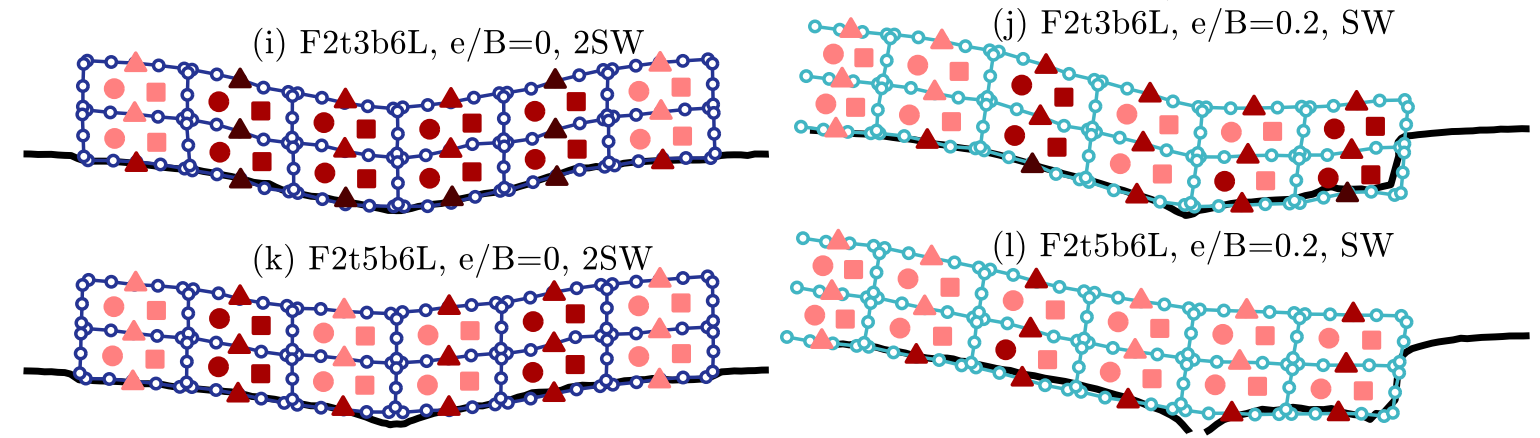

achoora (l) F2t5b6L, e/B=0.2, SW

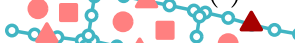

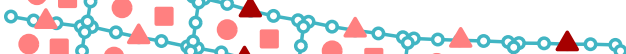

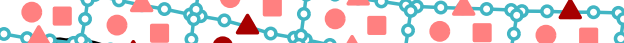
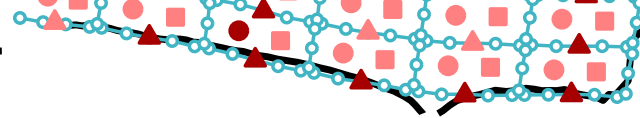

(n) F5t5b6L, e/B=0, SW, $1^{\text {st }}$ and $2^{\text {nd }}$ stories

(m) F5t5b6L, e/B=0, SW, $1^{\text {st }}$ and $2^{\text {nd }}$ stories

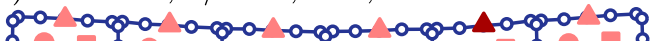
\$O \$O 8-a-o- $8-a-0-0-0-8-0-0-0-0-8-0-0-8$

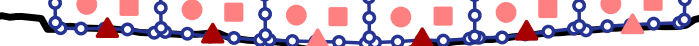

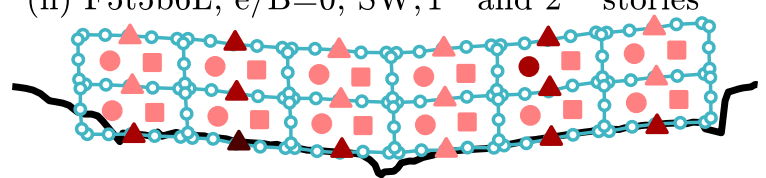

Fig. 13. Post-tunneling frame deformed shapes and level of damage (scale factor: 100); left column is dense sand tests, right column is loose sand tests.

sagging zone to spanning both sagging and hogging zones) significantly increases the maximum angular distortion; from low to medium levels based on Cook (1994) and Elkayam and Klar (2019), and from medium to high levels according to Boone (1996). Second, the short frame F2t3b3S in Figure 13(a)-(b) can be regarded as the left half of the long frame F2t3b6L in Figure 13(g)-(h). Results show that the short frame F2t3b3S with $e / B=0.5$ underwent significantly lower levels of 
distortion (low level for most panels) than the long frame $\mathrm{F} 2 \mathrm{t} 3 \mathrm{~b} 6 \mathrm{~L}$ with $e / B=0$ due to the effect of tilt for the eccentric configuration. These experimental results clearly display that the decrease of the transverse width $B$ has a positive effect on reducing framed building distortions (for both central and eccentric tunneling scenarios).

The effect of the width of the bay can be illustrated by comparing Figure 13(e)-(h). The results show that the external bays of the frame F2t3b3L (with larger bay width) experienced high levels of distortion in Figure 13(f) based on all approaches; this frame conformed closely to the ground movements. Frame distortion in Figure 13(e) tends to be similar to that in Figure 13(g), possibly due to the effect of averaging the $\beta$ values.

Interestingly, the decrease of soil relative density from $I_{d}=90 \%$ to $30 \%$ tends to increase the building distortion at a given $V_{l, t}$ because of higher values of $V_{l, s}$ (caused by greater levels of soil contraction for $I_{d}=30 \%$, refer to Figure 9), despite the increase of relative structure-soil stiffness. This can be seen in Figure 13(c-d), (e-f), (g-h), and (m-n); the stiffer the structure, the less the impact.

It is of interest to evaluate the efficiency of $D R$ in identifying the structures with the largest distortions. Figure 13(g), (h), (i), and (k) show that the maximum shear distortion of the central long frames was generally observed at the second and fifth bays (near where the maximum slope of the ground settlement trough occurs), whereas for the third and fourth bays, which were subjected to the largest relative deflections associated with $D R$, building distortions are relatively small. The underlying cause of these limitations related to the deflection ratio, as also pointed out by Finno et al. (2005), can be mechanically interpreted as follows. The experimental load-deflection tests illustrated that the structure deformations (i.e. their response to loadings) were shear-dominated. For Timoshenko beam theory, while bending-dominated structure settlement profiles naturally display a relative deflection, in a shear-dominated structure the relative deflection (of sagging/hogging portions) is due to the change in the value of internal shear forces (e.g. change in sign of the shear due to a concentrated force). Because diagonal strains $\epsilon_{x z}=\gamma / 2$ are proportional to the absolute value of the shear forces rather than the changes in forces, $D R$ is not as efficient as $\gamma \approx \beta$ to evaluate 
distortions of framed buildings.

Figure 13(g) and (i) show that the increase of building weight significantly increased the angular distortion for the third and fourth bays of the frame: from low to medium levels based on Cook (1994) and Elkayam and Klar (2019), and to a high level according to Boone (1996). At the same time, angular distortion is also observed to increase in the second and fifth bays according to Boone (1996); from medium to high. Furthermore, high levels of damage are observed for most parts of the foundation slab due to the redistribution of pressure along the soil-foundation interface.

Consistent with the short frame F2t3b3S, Figure 13(h) and (j) illustrate that the increase of building eccentricity decreased maximum shear distortions: from high to medium levels of damage for elevated slabs.

The increase of the thickness $t$ of building elements, which increased both stiffness and weight, resulted in the decrease of the structure distortions despite the increment in pressure beneath the building, as shown by comparing Figure 13(k) against (i), as well as (j) against (l).

Finally, to isolate the effects of building stiffness from changes in pressure, experimental results in Figure 13(k) and (m) can be compared since these two frames result in similar magnitudes of pressure beneath the building (refer to Table 4). As expected, results show that, for the given pressure, the increase of stiffness significantly reduced building distortions; from medium to low levels of damage for the second and fourth bays.

\section{Modification factor against relative soil-building stiffness}

Although deflection ratios $D R$ may not be fully representative when estimating the shear deformations of long frames, they are still valuable for evaluating the efficiency of design charts and interaction models in predicting the effects of soil-structure interaction.

Figure 14 displays the modification factors $M^{D R, s a g}$ and $M^{D R, h o g}$ at $V_{l, t}=1 \%$ and $2 \%$ against relative bending stiffness, $\rho_{\text {sag/hog }}$ (Mair, 2013) and $\eta_{\text {sag } / \text { hog }}$ (Franza et al., 2020), which were estimated using Equation (5) with $E I_{\text {exp }}$ and $G A_{s, e x p}$ as well as the values of $B_{s a g / h o g, g f}$ (i.e. based on greenfield displacements). Soil stiffness $E_{s}$ was estimated using the approach adopted by Farrell (2010), which consists of two steps: 1) a representative soil shear strain (considering stiffness 
degradation due to tunnel volume loss) is assessed at a mid-point between the surface and the tunnel crown for each tunnel-building interaction test; 2) soil stiffness $E_{S}$ is estimated using triaxial test provided by Zhao (2008) (for the same sand used in these tests). Using this approach, for greenfield tests, at $V_{l, t}=1-2 \%, E_{s}=68-45 \mathrm{MPa}$ for $I_{d}=90 \%$ and $E_{s}=34-21 \mathrm{MPa}$ for $I_{d}=30 \%$. For the extreme cases with the heaviest frame F5t5b6L, again at $V_{l, t}=1-2 \%, E_{s}=93-60 \mathrm{MPa}$ for $I_{d}=90 \%$, and $E_{s}=65-37 \mathrm{MPa}$ for $I_{d}=30 \%$. Data from all 22 tunnel-framed building interaction centrifuge tests presented in this paper are reported in Figure 14. Upper and lower envelopes suggested by Mair (2013) and Franza et al. (2020) are also included in Figure 14(a) and (b), respectively. In Figure 14(b), the envelopes for plane-strain conditions were selected.

The results in Figure 14(a) show that all the framed building data points are outside the envelopes when $\rho_{\text {sag } / \text { hog }}$ is estimated from the actual structure bending stiffness $E I_{\text {exp }}^{*}$, which for the considered frames is about a third of the bending stiffness suggested by Franzius et al. (2006) obtained when using parallel axis theorem. The structure stiffness relative to the soil was overestimated, and thus values of $M^{D R}$ are significantly underestimated. This is because this method neglects the impact of the global shear flexibility of the frame, which dominates the interaction problem for framed structures (with no bearing infill walls).

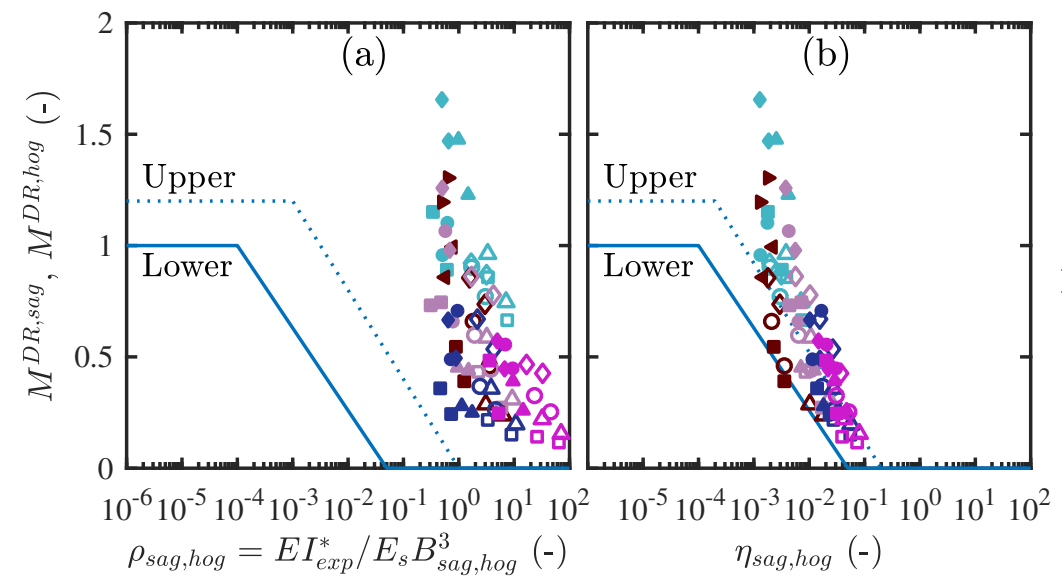

Color type $\quad$ Marker fill
$\Delta$ F2t3b3L $\Delta$ Sagging
$\Delta$ F2t3b3S $\Delta$ Hogging
$\Delta$ F2t3b6L
$\Delta$ F2t5b6L
$\Delta$ F5t5b6L
Marker style
$\Delta I_{d}=30 \%, e / B=0, \mathrm{SW}$
口 $I_{d}=30 \%, e / B=0.2 / 0.5, \mathrm{SW}$
○ $I_{d}=90 \%, e / B=0, \mathrm{SW}$
$\diamond I_{d}=90 \%, e / B=0,2 \mathrm{SW}$
$\triangleleft I_{d}=90 \%, e / B=0.5, \mathrm{SW}$
$\triangleright I_{d}=90 \%, e / B=0.5,2 \mathrm{SW}$

Fig. 14. Modification factors of $D R$ against relative stiffness $\left(V_{l, t}=1\right.$ and 2\%): (a) $\rho_{\text {sag } / \text { hog }}$ (Mair, 2013), and (b) $\eta_{\text {sag } / \text { hog }}$ (Franza et al., 2020).

Figure 14(b) shows that $\eta_{s a g / h o g}$ provides a better description of the relative structure-soil stiffness for framed buildings, supporting the robustness of the analytical interaction solutions of 
Franza et al. (2020). However, it is important to note that $\eta_{\text {sag } / \text { hog }}=\rho_{\text {sag } / \text { hog }}$ is obtained from Equation (4) by assuming $E I^{*}=E I_{E B, e q}^{*}=E t_{e q}^{3} / 12$. This confirms the reliability of the framework provided by Mair (2013) relating the equivalent (pure) bending stiffness $E I_{E B, e q}$ to the deflection ratio modification factor through $\rho_{\text {sag } / \text { hog }}$. Therefore, when estimating building stiffness, it is important to distinguish if the equivalent bending stiffness $E I_{E B, e q}$ is already decreased (to account for the shear flexibility) from the actual value of $E I$; for instance, $E I_{E B, e q}$ is obtained by using the column stiffness factor with the algebraic sum proposed by Goh and Mair (2014) rather than EI.

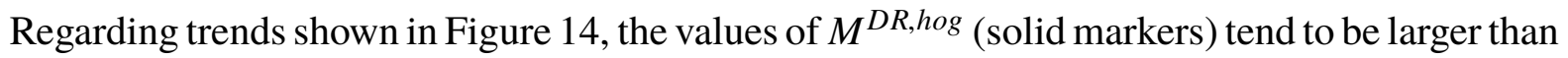
$M^{D R, s a g}$ (hollow markers) for all frames. The largest measured values of $M^{D R, s a g}$ are approximately 1.0, whereas the more flexible frames give $M^{D R, h o g}$ values greater than unity. This is possibly due to the effects of gap formation in the sagging zone (at $V_{l, t}=2 \%$ ) and frame embedment at its edges (with the formation or increase of a hogging settlement profile in the outer regions of the building).

To link angular distortion with relative soil-building stiffness, a logic similar to Son and Cording (2005) is followed by developing a modification factor chart to estimate $\beta$ from the slope of the greenfield surface settlement profile and a relative stiffness parameter that relates to the structure shear stiffness. First, for each bay of a given frame, the building angular distortion $\beta$ (estimated using Equation (6)) and average greenfield ground slope $\left(\overline{G S}=\Delta U_{z, g f, \max } / b_{b a y}\right.$, where $\Delta U_{z, g f, \max }$ is the differential settlement of the portion of greenfield surface settlement trough corresponding to $\left.b_{\text {bay }}\right)$ are calculated. Then, the maximum experimental angular distortion among all bays $\beta_{\max }$ is normalized by the maximum greenfield slope corresponding to the building $\left(\overline{G S}_{\text {max }}\right)$ to obtain a modification factor of angular distortion, $M^{\beta}$ :

$$
M^{\beta}=\frac{\beta_{\text {max }}}{\overline{G S}_{\max }}
$$

For instance, $M^{\beta}=1$ for a fully flexible structure deforming purely in shear according to the greenfield settlement trough with local tilt $\omega=0$. The modification factor of angular distortion is then related to the relative soil-building shear stiffness, estimated as

$$
\kappa=\frac{E_{s} B}{G A_{s}^{*}}=\frac{E_{s} B L}{G A_{s}}
$$


where $G A_{s}^{*}$ is the building shear stiffness per meter run (i.e. $G A_{s}$ normalized by the building length $L$ in the longitudinal direction of the tunnel).

Figure 15 presents the relationship between $M^{\beta}$ and $\kappa$ (in log scale) at $V_{l, t}=1$ and $2 \%$ (numbers beneath markers indicate $V_{l, t}$ ). To highlight the effects of soil density and building transverse width, a color scheme was adopted to distinguish between soil density and the value of the ratio $B / i$, where $i$ is the distance to the inflection point of the greenfield settlement trough. Data from central $(e / B=0)$ and eccentric $(e / B>0)$ structures are plotted in Figures 15(a) and (b), respectively. Several trends can be gleaned from Figure 15: $M^{\beta}$ tends to increase linearly with an increase of relative soil-building shear stiffness (which agrees with Son and Cording (2005)); a decrease in building width ratio $B / i$ decreases $M^{\beta}$; and an increase of building eccentricity $e / B$ reduces $M^{\beta}$ (because of the building tilt, which is in agreement with Figure 13). Finally, an increase of building weight also results in a higher $M^{\beta}$ (compare hollow to solid markers), partially because of greater ground deformations transmitted to the heavier buildings in cases where gap formation was less significant. Upper and lower envelopes for the modification factors of building angular distortions are also suggested in Figure 15.
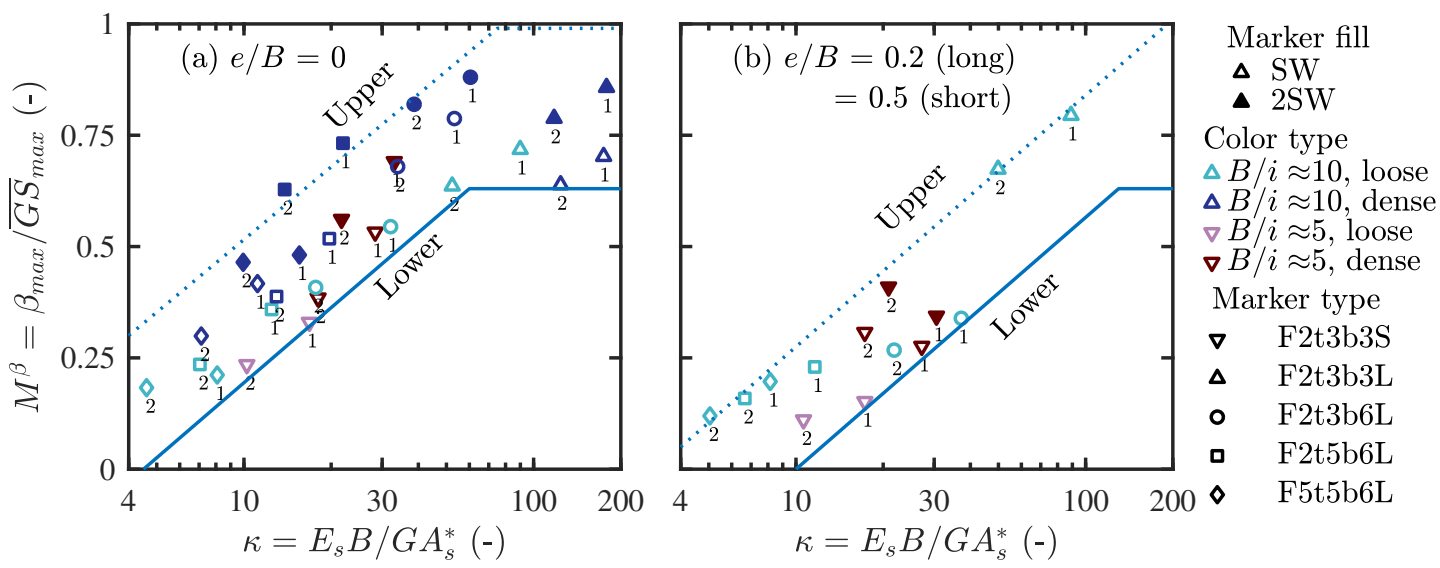

Fig. 15. Modification factors of $\beta$ against relative soil-building shear stiffness (numbers beneath markers indicate $\left.V_{l, t}\right)$.

\section{CONCLUSIONS}

A comprehensive study on the response of framed buildings on raft foundations to tunneling was presented, including data from a unique set of centrifuge tests. The tunneling-induced displacements 
of the framed buildings and the underlying soil were analyzed, and the framed structure deformations were studied using deflection ratios, angular distortions, and strain-based damage categories. The following conclusions can be drawn from the work.

- Comparison of settlement profiles demonstrated that the settlements of equivalent isotropic plate models (with a bending dominated deformation mode) do not always adequately match those from framed building models, missing important features mainly with respect to the shape of the foundation settlement.

- Generally, for both loose and dense soil, a greater building weight and larger transverse width increase the tunneling-induced framed building distortions, whereas a larger building eccentricity (facilitating building tilt) and higher stiffness tend to reduce frame distortions.

- For a given tunnel volume loss (representative of the performance of tunneling operations), the relative density of dry sands impacted the level of settlement of the ground surface and, consequently, the distortions of the frames. Loose sands have greater potential for building distortions than dense sands at a given tunnel volume loss. On the contrary, for a given soil volume loss at the ground surface, buildings experienced similar levels of settlement and distortion for varying sand densities, with loose sand tests giving slightly smaller distortions.

- Experimental data of deflection ratio modification factors agreed with existing charts; in particular, experimental results confirmed that the bending and shear stiffness of framed buildings may be directly estimated by modeling load-deflection tests.

- A modification factor was suggested to link framed building angular distortion to relative soilbuilding shear stiffness, in which interesting linear trends were highlighted; upper and lower envelopes for preliminary damage assessment considering building eccentricity were suggested.

- Several key aspects were highlighted from the work which have implications for engineering practice. First, the need to monitor/evaluate framed building displacements at multiple elevations to better estimate deformations and damage was confirmed; foundation level deformations may be greater than within the superstructure. Framed buildings on raft foundations will likely have 
a shear dominated deformation mode. Framed structures centrally located above a tunnel will have the greatest potential for distortions compared to eccentric cases. When possible, to better estimate the distortions of frames, shear strains should be evaluated using the displacements of the corners of bays/panels rather than using a deflection ratio based evaluation.

In this paper, the considered scenarios are limited to a tunnel with constant cover-to-diameter ratio constructed in dry sand beneath an elastic framed building with a raft foundation. Future work could investigate the effects of tunnel relative depth, water table, foundation type, foundation embedment, building skew, and nonlinear building behavior.

\section{DATA AVAILABILITY}

Data are available from the authors on request.

\section{ACKNOWLEDGEMENTS}

This project has received funding from the European Union's Horizon 2020 research and innovation programme under the Marie Sklodowska-Curie grant agreement No 793715. The first author also recognizes the financial support provided by the China Scholarship Council (CSC) and the University of Nottingham, UK. 


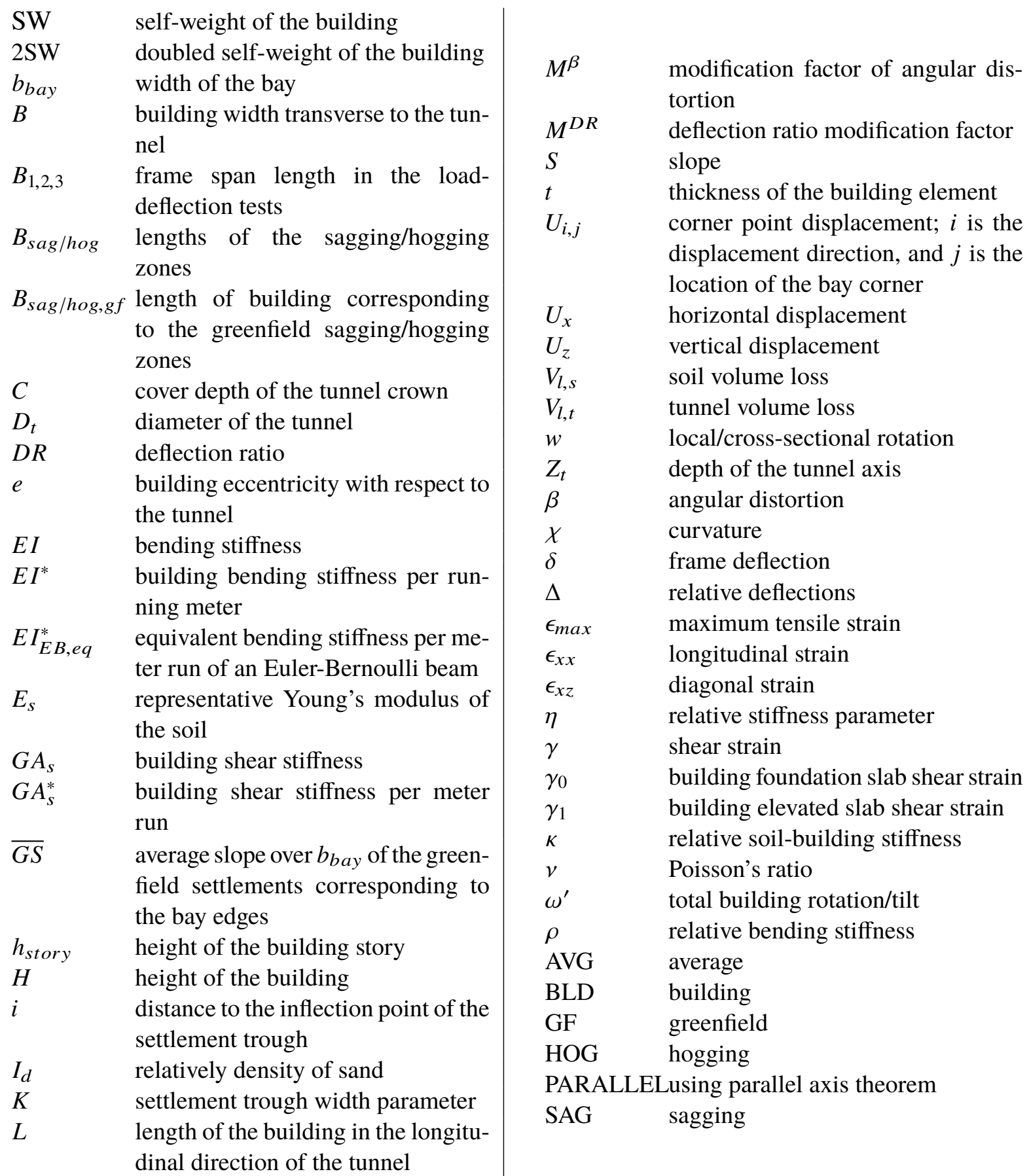




\section{REFERENCES}

Bilotta, E., Paolillo, A., Russo, G., and Aversa, S. (2017). "Displacements induced by tunnelling under a historical building." Tunn. Undergr. Sp. Technol., 61, 221-232.

Boldini, D., Losacco, N., Bertolin, S., and Amorosi, A. (2018). "Finite element modelling of tunnellinginduced displacements on framed structures." Tunn. Undergr. Sp. Technol., 80(April), 222-231.

Boone, S. J. (1996). “Ground-movement-related building damage.” J. Geotech. Eng., 122(11), 886-896.

Boscardin, M. D. and Cording, E. J. (1989). "Building response to excavation-induced settlement." $J$. Geotech. Eng., 115(1), 1-21.

Cook, D. (1994). "Studies of settlement and crack damage in old and new facades." Proc., 3rd Int. Masonry Conf., London, England, Vol. 6, 203-211.

Deck, O. and Singh, A. (2012). "Analytical model for the prediction of building deflections induced by ground movements." Int. J. Numer. Anal. Met., 36(1), 62-84.

Elkayam, I. and Klar, A. (2019). "Nonlinear elastoplastic formulation for tunneling effects on superstructures." Can. Geotech. J., 56(7), 956-969.

Farrell, R. (2010). "Tunnelling in sands and the response of buildings." Ph.D. Thesis, Cambridge Univ.

Farrell, R., Mair, R., Sciotti, A., and Pigorini, A. (2014). "Building response to tunnelling." Soils Found., 54(3), 269-279.

Finno, R. J., Voss, F. T., Rossow, E., and Blackburn, J. T. (2005). "Evaluating damage potential in buildings affected by excavations." J. Geotech. Geoenviron. Eng., 131(10), 1199-1210.

Franza, A., Acikgoz, S., and DeJong, M. J. (2020). "Timoshenko beam models for the coupled analysis of building response to tunnelling." Tunn. Undergr. Sp. Technol., 96(October 2019), 103160.

Franza, A. and DeJong, M. J. (2019). "Elastoplastic solutions to predict tunnelling-induced load transfer and deformation mechanisms of surface structures." J. Geotech. Geoenviron. Eng., 145, 04019007.

Franza, A. and Marshall, A. M. (2018). "Centrifuge modeling study of the response of piled structures to tunneling.” J. Geotech. Geoenviron. Eng., 144(2), 04017109.

Franza, A., Marshall, A. M., and Zhou, B. (2019a). "Greenfield tunnelling in sands: the effects of soil density and relative depth." Géotechnique, 69(4), 297-307.

Franza, A., Ritter, S., and Dejong, M. J. (2019b). "Continuum solutions for tunnel-building interaction and a modified framework for deformation prediction." Géotechnique, 70(2), 108-122.

Franzius, J., Potts, D., and Burland, J. (2006). "The response of surface structures to tunnel construction." Proc. Inst. Civ. Eng. Geotech. Eng., 159(1), 3-17.

Fu, J., Yu, Z., Wang, S., and Yang, J. (2018). "Numerical analysis of framed building response to tunnelling induced ground movements." Eng. Struct., 158, 43-66.

Goh, K. H. and Mair, R. J. (2014). "Response of framed buildings to excavation-induced movements." Soils Found., 54(3), 250-268.

Haji, T. K., Marshall, A. M., and Tizani, W. (2018). “A cantilever approach to estimate bending stiffness of buildings affected by tunnelling." Tunn. Undergr. Sp. Technol., 71, 47-61.

Losacco, N., Callisto, L., and Burghignoli, A. (2016). "Soil-structure interaction due to tunnelling in soft ground, an equivalent solid approach." Int. Conf. Struct. Anal. Hist. Constr., Belgium, Leuven, K. Van Balen and E. Verstrynge, eds., CRC Press, 495-501 (sep). 
Mair, R. (2013). "Tunnelling and deep excavations: ground movements and their effects." Proc. 15th Eur. Conf. Soil Mech. Geotech. Eng. - Geotech. Hard Soils - Weak Rocks (Part 4), A. Anagnostopoulos, M. Pachakis, and C. Tsatsanifos, eds., Amsterdam, the Netherlands, IOS Press, 39 - 70.

Mair, R. J., Taylor, R. N., and Burland, J. B. (1996). "Prediction of ground movements and assessment of risk of building damage due to bored tunnelling." Proc. Int. Symp. Geotech. Aspects Undergr. Constr. Soft Ground, R. J. Mair and R. N. Taylor, eds., London, United Kingdom, Balkema, Rotterdam, 713-718.

Maleki, M., Sereshteh, H., Mousivand, M., and Bayat, M. (2011). "An equivalent beam model for the analysis of tunnel-building interaction.” Tunn. Undergr. Sp. Technol., 26(4), 524-533.

Marshall, A. M., Farrell, R., Klar, A., and Mair, R. (2012). "Tunnels in sands: the effect of size, depth and volume loss on greenfield displacements." Géotechnique, 62(5), 385-399.

Namazi, E. and Mohamad, H. (2013). "Assessment of Building Damage Induced by Three-Dimensional Ground Movements." J. Geotech. Geoenviron. Eng., 139(4), 608-618.

Pickhaver, J., Burd, H., and Houlsby, G. (2010). "An equivalent beam method to model masonry buildings in 3D finite element analysis." Comput. Struct., 88(19), 1049-1063.

Potts, D. and Addenbrooke, T. (1997). "A structure's influence on tunnelling induced ground movements." Geotech. Eng., 125, 109-125.

Ritter, S., Giardina, G., DeJong, M. J., and Mair, R. J. (2017). "Centrifuge modelling of building response to tunnel excavation." Int. J. Phys. Model. Geotech., 18(3), 146-161.

Ritter, S., J. Dejong, M., Giardina, G., Franza, A., and J. Mair, R. (2020). "Building deformation caused by tunnelling: centrifuge modelling." J. Geotech. Geoenviron. Eng. in press, DOI: 10.1061/(ASCE)GT.19435606.0002223 .

Son, M. (2015). "Response analysis of nearby structures to tunneling-induced ground movements in sandy soils." Tunn. Undergr. Sp. Technol., 48, 156-169.

Son, M. and Cording, E. J. (2005). "Estimation of building damage due to excavation-induced ground movements." J. Geotech. Geoenviron. Eng., 131(2), 162-177.

Son, M. and Cording, E. J. (2007). "Evaluation of building stiffness for building response analysis to excavation-induced ground movements." J. Geotech. Geoenviron. Eng., 133(8), 995-1002.

Taylor, R. N. (1995). Geotechnial centrifuge technology. Blackie Academic \& Professional, London.

Vorster, T. E. B., Klar, A., Soga, K., and Mair, R. J. (2005). "Estimating the effects of tunneling on existing pipelines." J. Geotech. Geoenviron. Eng., 131(11), 1399-1410.

White, D., Take, W., and Bolton, M. (2003). "Soil deformation measurement using particle image velocimetry (PIV) and photogrammetry." Géotechnique, 53(7), 619-631.

Xu, J., Marshall, M. A., Franza, A., Boldini, D., Amorosi, A., and DeJong, J. M. (2019). "The response of framed buildings on raft foundations to tunnelling: a centrifuge and numerical modelling study." 17th Eur. Conf. Soil Mech. Geotech. Eng., Reykjavik, Iceland, Vol. 1, 1-8.

Zhao, Y. (2008). "In situ soil testing for foundation performance prediction.” Ph.D. Thesis, Cambridge Univ. Zhou, B. (2014). “Tunnelling-induced ground displacements in sand.” Ph.D. Thesis, Univ. of Nottingham. 


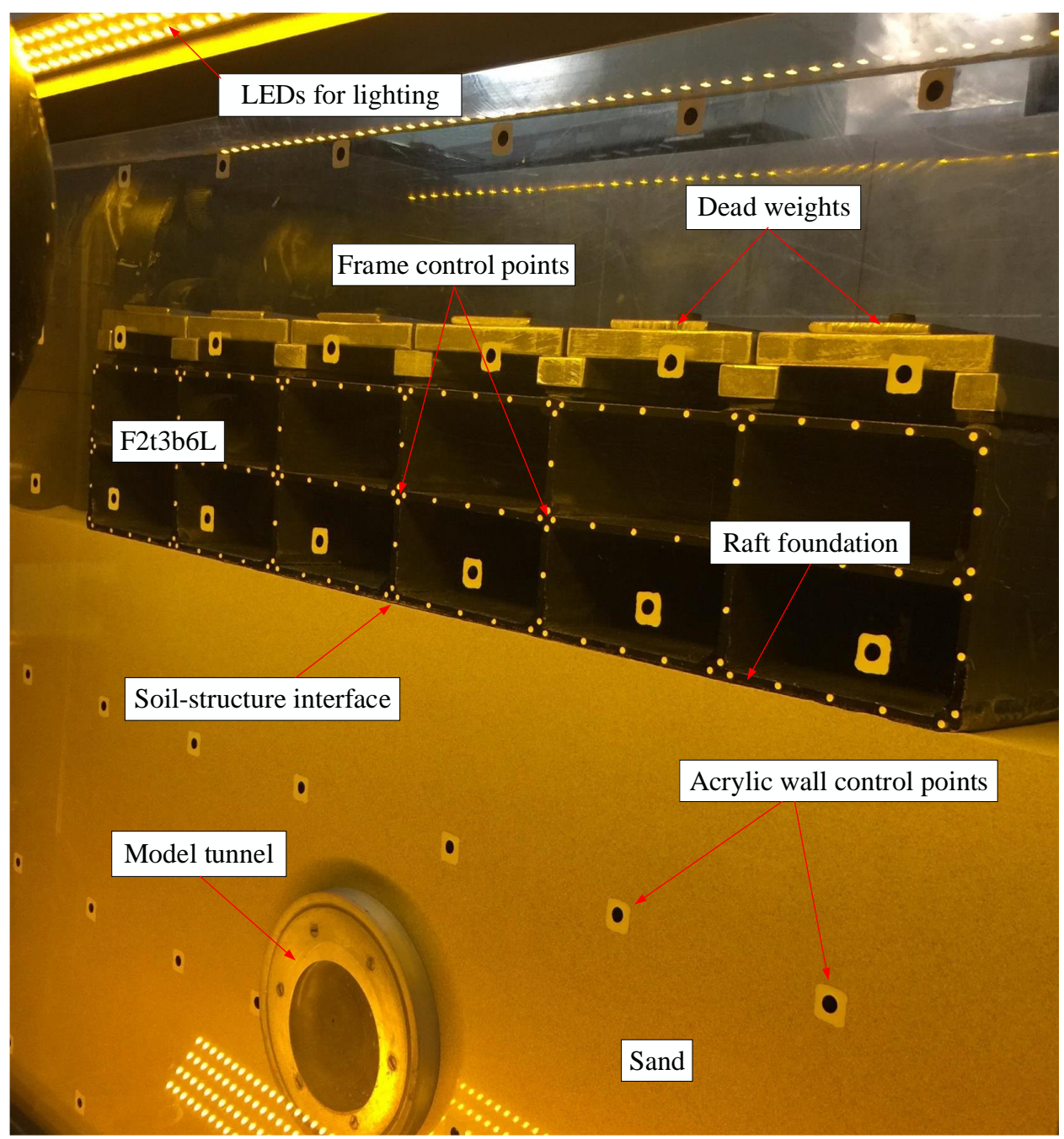

Fig. S1. Set up of centrifuge model with frame F2t3b6L. 


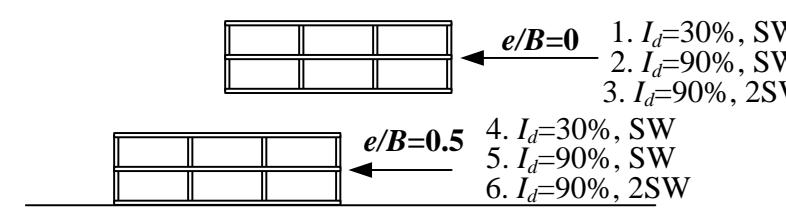

F2t3b3S (6 tests)
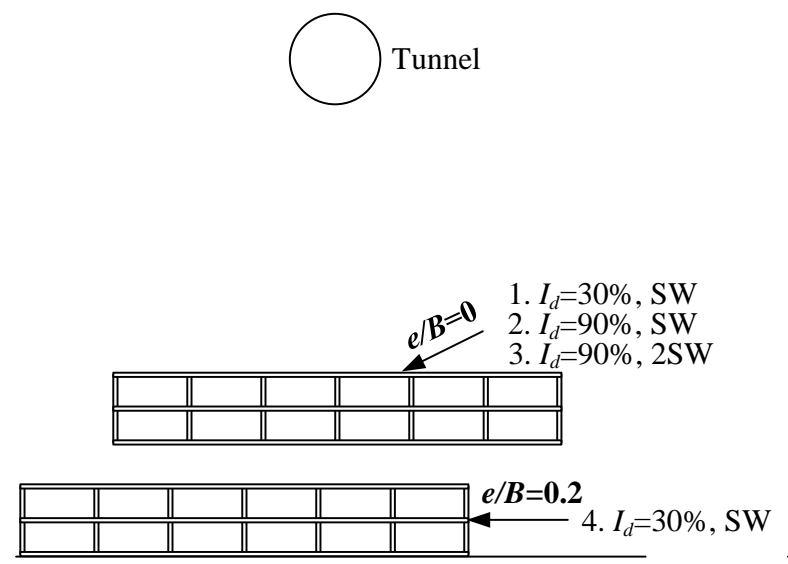

F2t3b6L (4 tests) /F2t5b6L (4 tests)<smiles>Cl[C]1CCCCCC1</smiles>
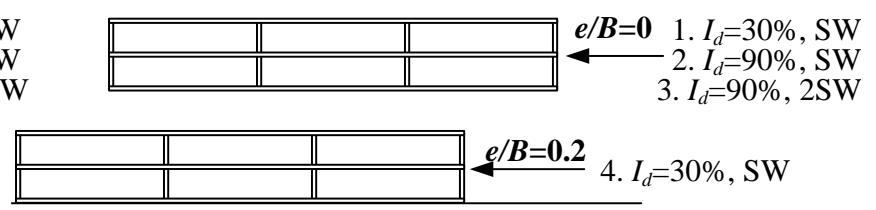

F2t3b3L (4 tests)
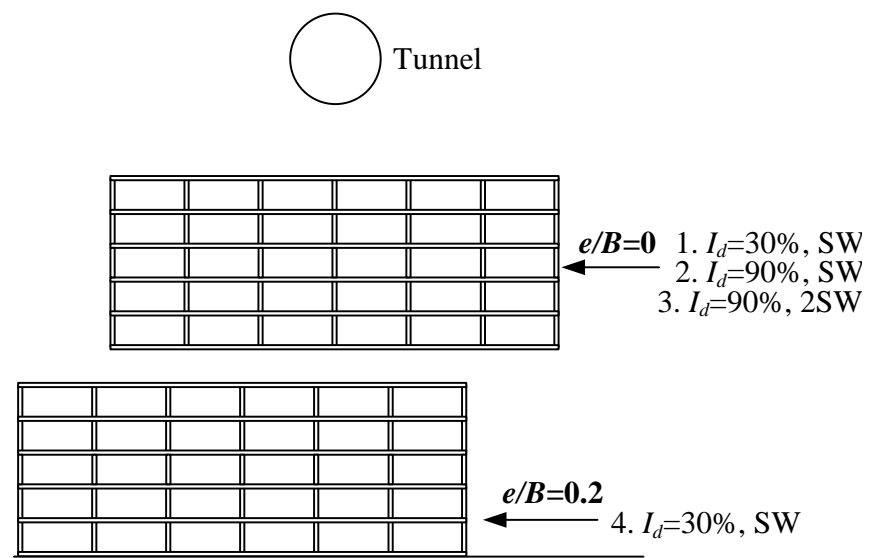

F5t5b6L (4 tests)

Fig. S2. Framed building model configurations and testing plan.
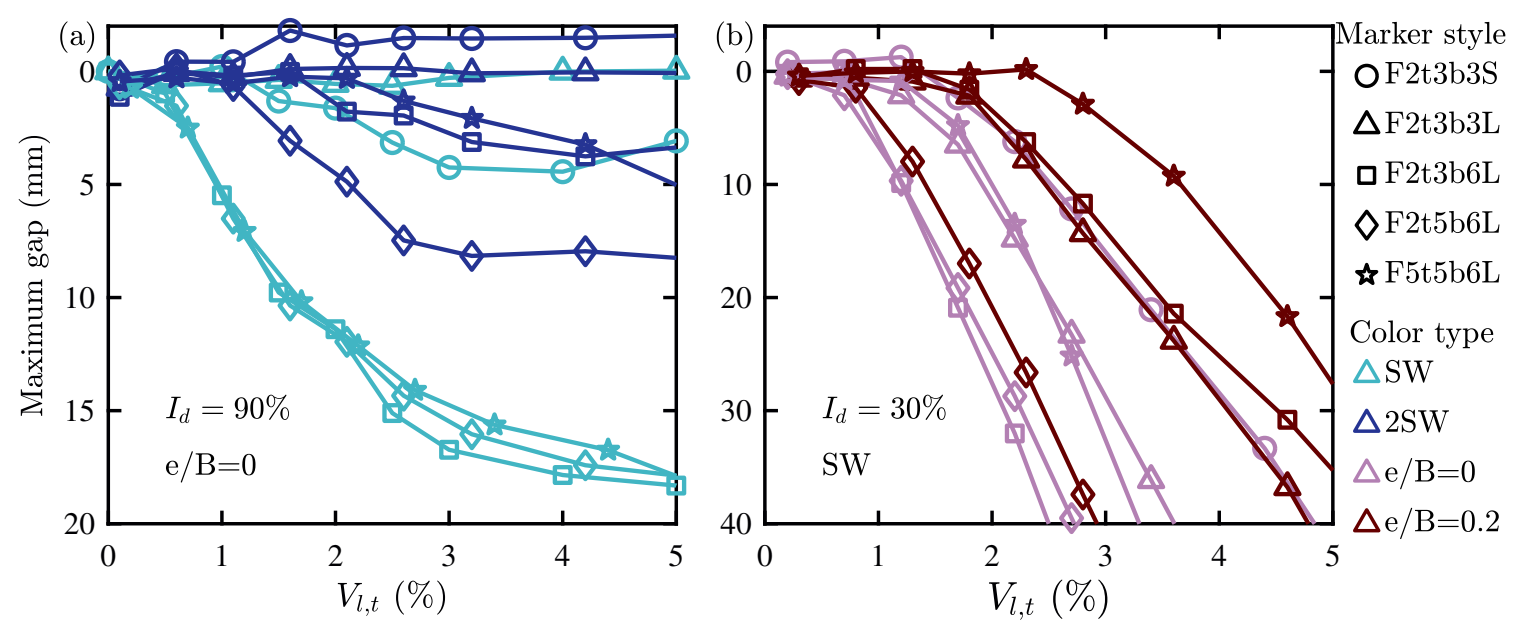

Fig. S3. Maximum gap height against tunnel volume loss. 
TABLE S1. Experimental results of bending and shear stiffness (per running meter) of building models (at model scale).

\begin{tabular}{|c|c|c|c|c|c|c|c|}
\hline Frame & Tests $(\dagger)$ & $\begin{array}{l}E I_{\text {exp }}^{*} \\
(\mathrm{Nm})\end{array}$ & $\begin{array}{c}E I_{\text {exp,avg }}^{*} \\
(\mathrm{Nm})\end{array}$ & $\begin{array}{c}E I_{\text {parallel }}^{*} \\
(\mathrm{Nm})\end{array}$ & $\begin{array}{c}G A_{s, \exp }^{*} \\
(\mathrm{~N} / \mathrm{m})\end{array}$ & $\begin{array}{c}G A_{s, \text { exp, avg }}^{*} \\
(\mathrm{~N} / \mathrm{m})\end{array}$ & $\delta_{\text {shear }} / \delta_{\text {bending }}$ \\
\hline \multirow{6}{*}{ F2t3b6L } & $1 \& 2$ & $1.8 \mathrm{E}+05$ & \multirow{6}{*}{$1.9 \mathrm{E}+05$} & \multirow{6}{*}{$6.6 \mathrm{E}+05$} & $6.2 \mathrm{E}+05$ & \multirow{6}{*}{$6.2 \mathrm{E}+05$} & \multirow{6}{*}{18.0} \\
\hline & $1 \& 3$ & $2.1 \mathrm{E}+05$ & & & $6.2 \mathrm{E}+05$ & & \\
\hline & $1 \& 4$ & $2.0 \mathrm{E}+05$ & & & $6.2 \mathrm{E}+05$ & & \\
\hline & $2 \& 3$ & $2.1 \mathrm{E}+05$ & & & $6.2 \mathrm{E}+05$ & & \\
\hline & $2 \& 4$ & $2.0 \mathrm{E}+05$ & & & $6.2 \mathrm{E}+05$ & & \\
\hline & $3 \& 4$ & $1.8 \mathrm{E}+05$ & & & $6.2 \mathrm{E}+05$ & & \\
\hline \multirow{3}{*}{$\mathrm{F} 2 \mathrm{t} 5 \mathrm{~b} 6 \mathrm{~L}$} & $1 \& 2$ & $2.9 \mathrm{E}+05$ & \multirow{3}{*}{$2.8 \mathrm{E}+05$} & \multirow{3}{*}{$9.7 \mathrm{E}+05$} & $1.9 \mathrm{E}+06$ & & \multirow{3}{*}{8.2} \\
\hline & $1 \& 3$ & $2.9 \mathrm{E}+05$ & & & $1.9 \mathrm{E}+06$ & $1.9 \mathrm{E}+06$ & \\
\hline & $2 \& 3$ & $2.4 \mathrm{E}+05$ & & & $1.9 \mathrm{E}+06$ & & \\
\hline \multirow{3}{*}{ F5t5b6L } & $1 \& 2$ & $3.1 \mathrm{E}+06$ & \multirow{3}{*}{$3.1 \mathrm{E}+06$} & \multirow{3}{*}{$8.5 \mathrm{E}+06$} & $3.9 \mathrm{E}+06$ & & \multirow{3}{*}{44.5} \\
\hline & $1 \& 3$ & $3.1 \mathrm{E}+06$ & & & $3.9 \mathrm{E}+06$ & $3.9 \mathrm{E}+06$ & \\
\hline & $2 \& 3$ & $3.1 \mathrm{E}+06$ & & & $3.9 \mathrm{E}+06$ & & \\
\hline $\mathrm{F} 2 \mathrm{t} 3 \mathrm{~b} 3 \mathrm{~L}$ & 3 & $1.9 \mathrm{E}+05$ & - & $6.6 \mathrm{E}+05$ & $2.1 \mathrm{E}+05$ & - & 51.7 \\
\hline
\end{tabular}

(†) \#1: 3-point bending with $B_{1}$; \#2: 3-point bending with $B_{2}$; \#3: 4-point bending with $B_{1}$; \#4: cantilever.

TABLE S2. Maximum building angular distortion $(\gamma=\beta)$ at $V_{l, t}=2 \%$.

\begin{tabular}{llllllll}
\hline Test label & $\begin{array}{l}\mathrm{Bay}_{1} \\
(\%)\end{array}$ & $\begin{array}{l}\mathrm{Bay}_{2} \\
(\%)\end{array}$ & $\begin{array}{l}\mathrm{Bay}_{3} \\
(\%)\end{array}$ & $\begin{array}{l}\mathrm{Bay}_{4} \\
(\%)\end{array}$ & $\begin{array}{l}\mathrm{Bay}_{5} \\
(\%)\end{array}$ & $\begin{array}{l}\text { Bay6 } \\
(\%)\end{array}$ & $\begin{array}{l}\text { Soil } \\
\text { relative density }\end{array}$ \\
\hline F2t3b3S_eB0.5_SW & 0.06 & 0.02 & 0.09 & - & - & - & \\
F2t3b3S_eB0_SW & 0.10 & 0.02 & 0.12 & - & - & - & \\
F2t3b3L_eB0_SW & 0.14 & 0.00 & 0.15 & - & - & - & \\
F2t3b6L_eB0_SW & 0.08 & 0.15 & 0.03 & 0.06 & 0.20 & 0.10 & $I_{d}=90 \%$ \\
F2t3b6L_eB0_2SW & 0.08 & 0.23 & 0.15 & 0.16 & 0.25 & 0.09 & \\
F2t5b6L_eB0_2SW & 0.09 & 0.17 & 0.08 & 0.11 & 0.19 & 0.08 & \\
F5t5b6L_eB0_SW & 0.05 & 0.07 & 0.05 & 0.01 & 0.09 & 0.05 & \\
\hline F2t3b3S_eB0.5_SW & 0.07 & 0.02 & 0.08 & - & - & - & \\
F2t3b3S_eB0_SW & 0.11 & 0.03 & 0.16 & - & - & - & \\
F2t3b3L_eB0_SW & 0.30 & 0.03 & 0.33 & - & - & - & \\
F2t3b6L_eB0_SW & 0.13 & 0.24 & 0.09 & 0.08 & 0.28 & 0.15 & $I_{d}=30 \%$ \\
F2t3b6L_eB0.2_SW & 0.04 & 0.02 & 0.16 & 0.12 & 0.14 & 0.19 & \\
F2t5b6L_eB0.2_SW & 0.00 & 0.07 & 0.11 & 0.03 & 0.09 & 0.11 & \\
F5t5b6L_eB0_SW & 0.07 & 0.12 & 0.07 & 0.06 & 0.13 & 0.06 & \\
\hline
\end{tabular}




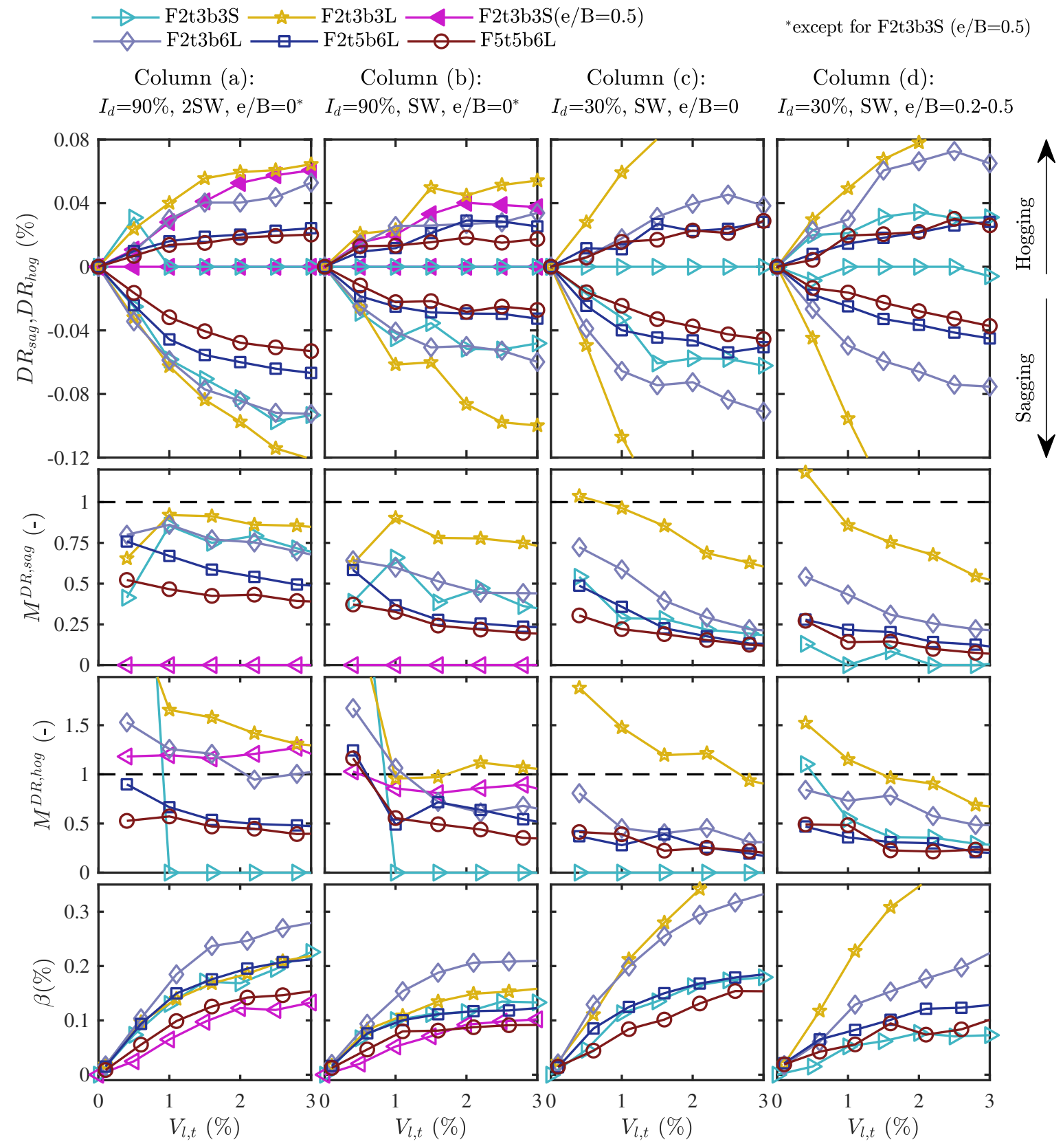

Fig. S4. Building distortion parameters against tunnel volume loss. 


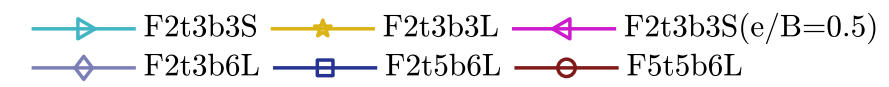

(a):

(b):

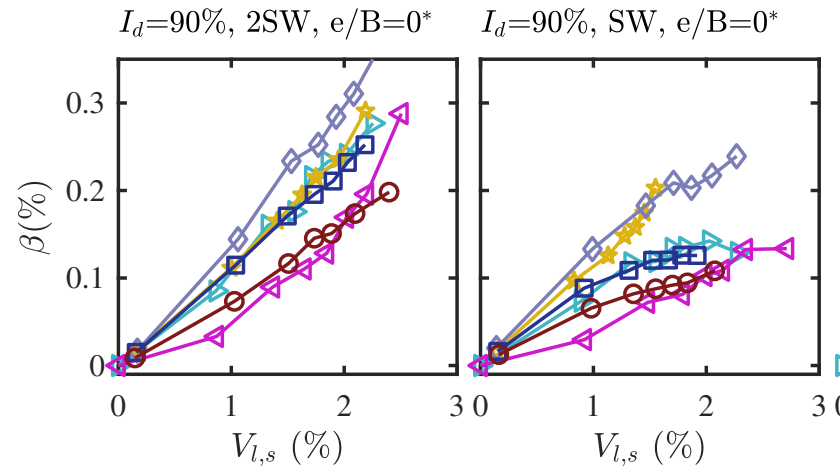

${ }^{*}$ except for $\mathrm{F} 2 \mathrm{t} 3 \mathrm{~b} 3 \mathrm{~S}(\mathrm{e} / \mathrm{B}=0.5)$

(c):

(d):

$I_{d}=30 \%, \mathrm{SW}, \mathrm{e} / \mathrm{B}=0 \quad I_{d}=30 \%, \mathrm{SW}, \mathrm{e} / \mathrm{B}=0.2-0.5$

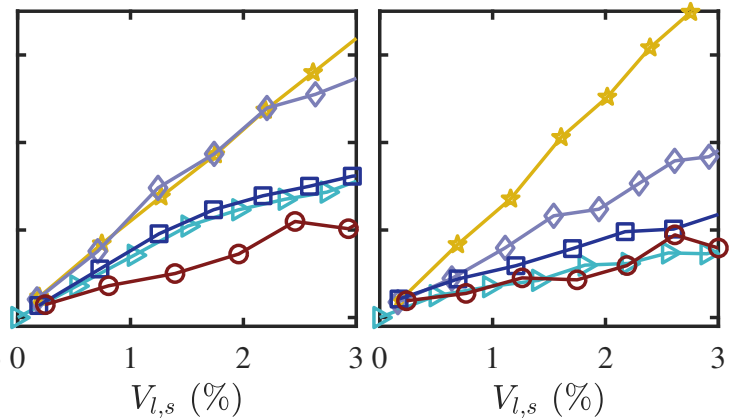

Fig. S5. Building angular distortion against soil volume loss. 